\title{
Setbacks in the Clinical Development of TRPV1 Antagonists: What Next?
}

Narender R. Gavva*

Department of Neuroscience, Amgen, Inc., Thousand Oaks, CA, USA

Abstract: TRPV1 antagonists have been considered as potential treatments for pain associated with inflammatory diseases and cancer. During Phase I clinical trials with AMG 517, a highly selective TRPV1 antagonist, we found that TRPV1 blockade elicits marked, but reversible, and generally plasma concentration-dependent hyperthermia. Furthermore, in a Phase Ib study, AMG 517 administered after molar extraction (a surgical cause of acute pain) elicited long-lasting hyperthermia with maximal body temperature surpassing $40^{\circ} \mathrm{C}$, suggesting that TRPV1 blockade elicits undesirable hyperthermia in susceptible individuals.

Since TRPV1 blockade elicited hyperthermia is a major hurdle, we investigated the possibility of eliminating hyperthermia while maintaining antihyperalgesia by two approaches: i) peripheral restriction of TRPV1 antagonists, ii) characterization of TRPV1 modulators that exhibit differential pharmacology. Results from the preclinical studies of both approaches will be discussed.

*Address correspondence to this author at the Department of Neuroscience, Amgen Inc., Thousand Oaks, CA, USA; Tel: (805)447-0607; E-mail: ngavva@amgen.com 
Presented at the $2^{\text {nd }}$ annual Pain therapeutics summit, Oct 6-7, 2008, New Brunswick, NJ

\title{
Setbacks in the Clinical Development of TRPV1 Antagonists: What Next?
}

\author{
Narender R. Gavva Ph.D. \\ Department of Neuroscience
}




\section{Outline}

- Introduction

- Functional assays

- AMG 517 preclinical studies

- TRPV1 antagonism - hyperthermia

- Mechanisms of hyperthermia

- AMG 517 clinical trial results

- Peripherally restricted antagonists - hyperthermia

- Differential pharmacology of antagonists - no hyperthermia

- Conclusions 


\section{TRP channels are integrators of multiple noxious stimuli}

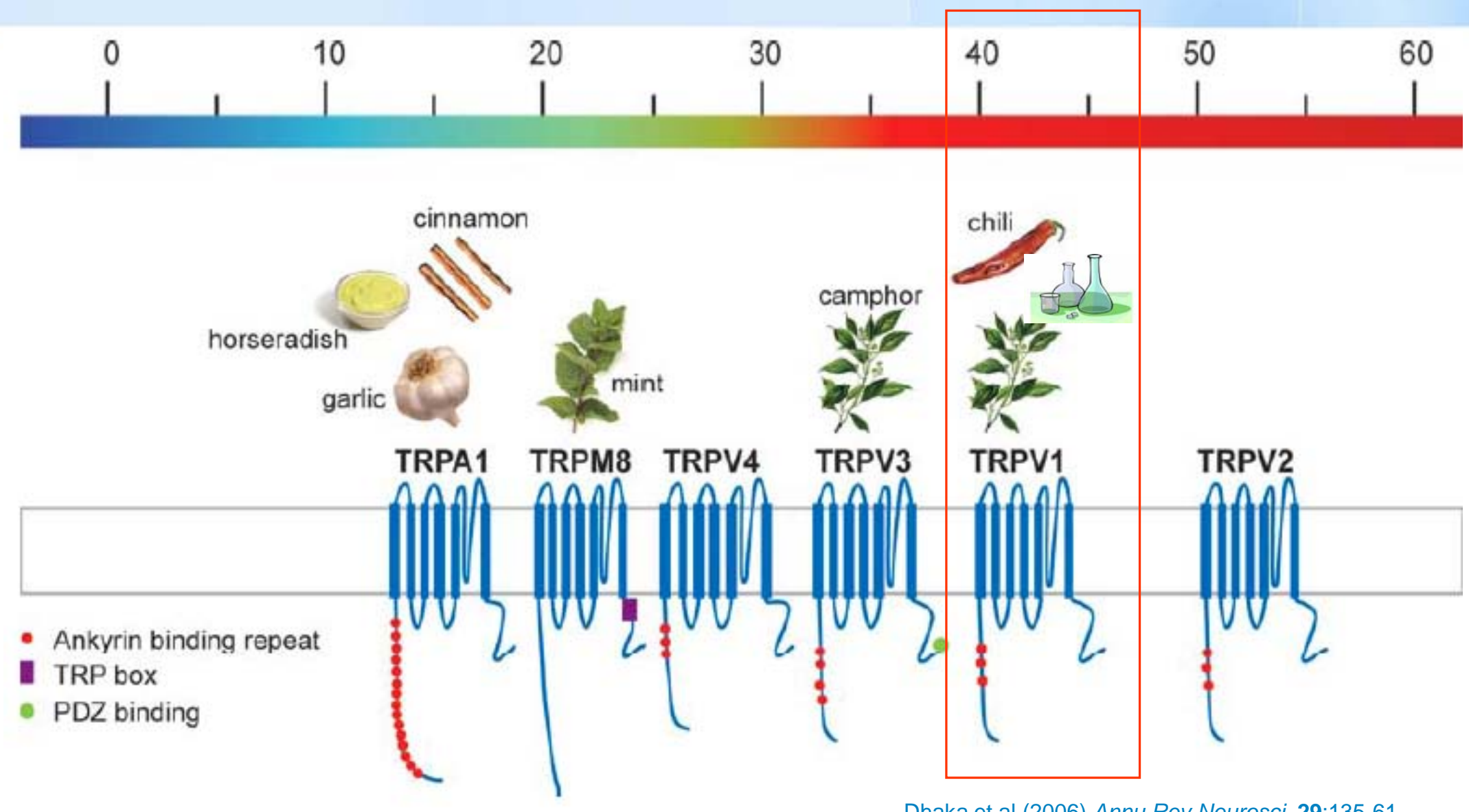

Dhaka et al (2006) Annu Rev Neurosci. 29:135-61

- $\quad$ Agonists of TRPA1 and TRPV1 cause pain in humans and pain behavior in rodents 


\section{TRPV1: A polymodal detector of painful stimuli}

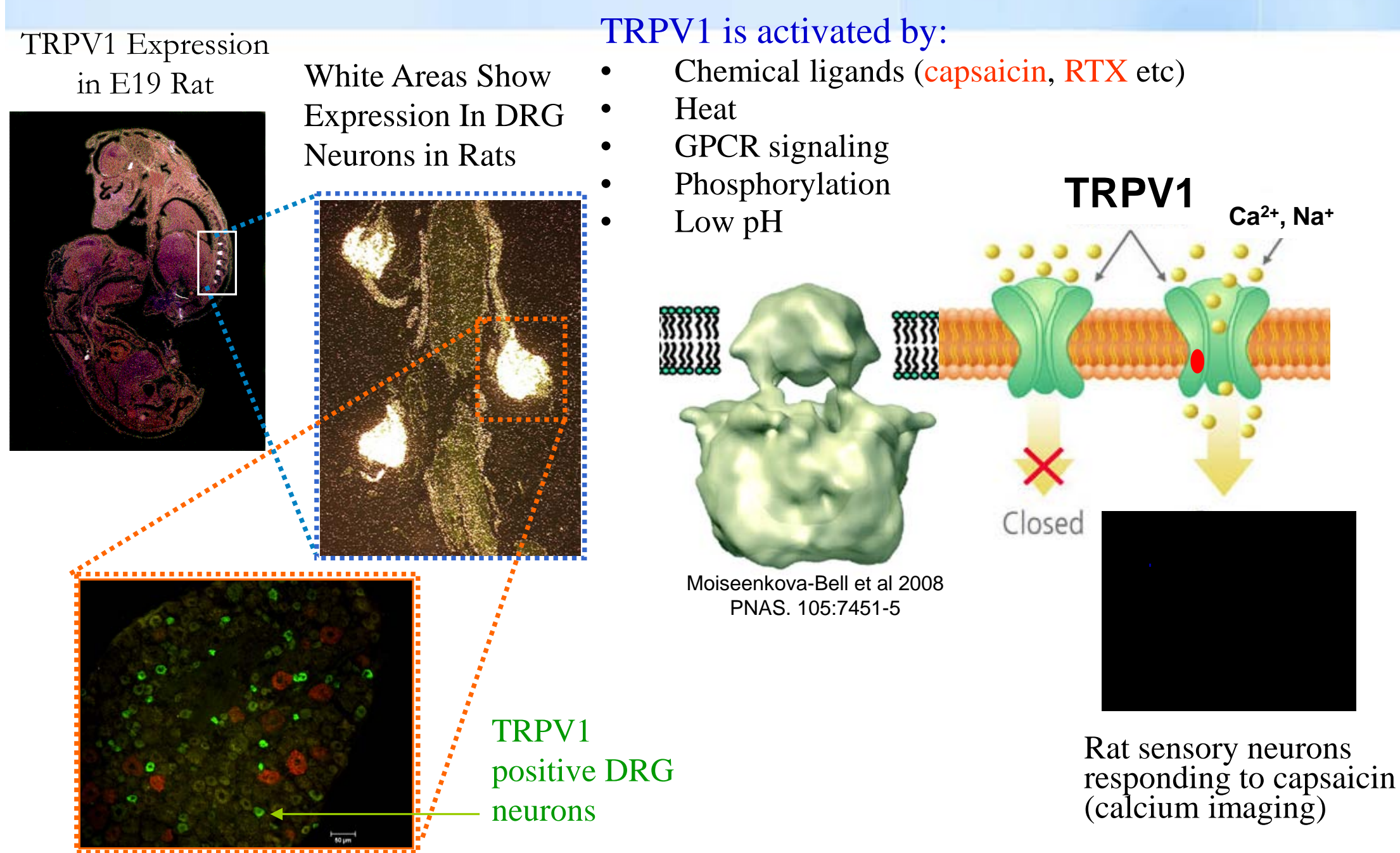




\section{TRPV1 - Target validation}

TRPV1 knockout mice show reduced thermal hyperalgesia
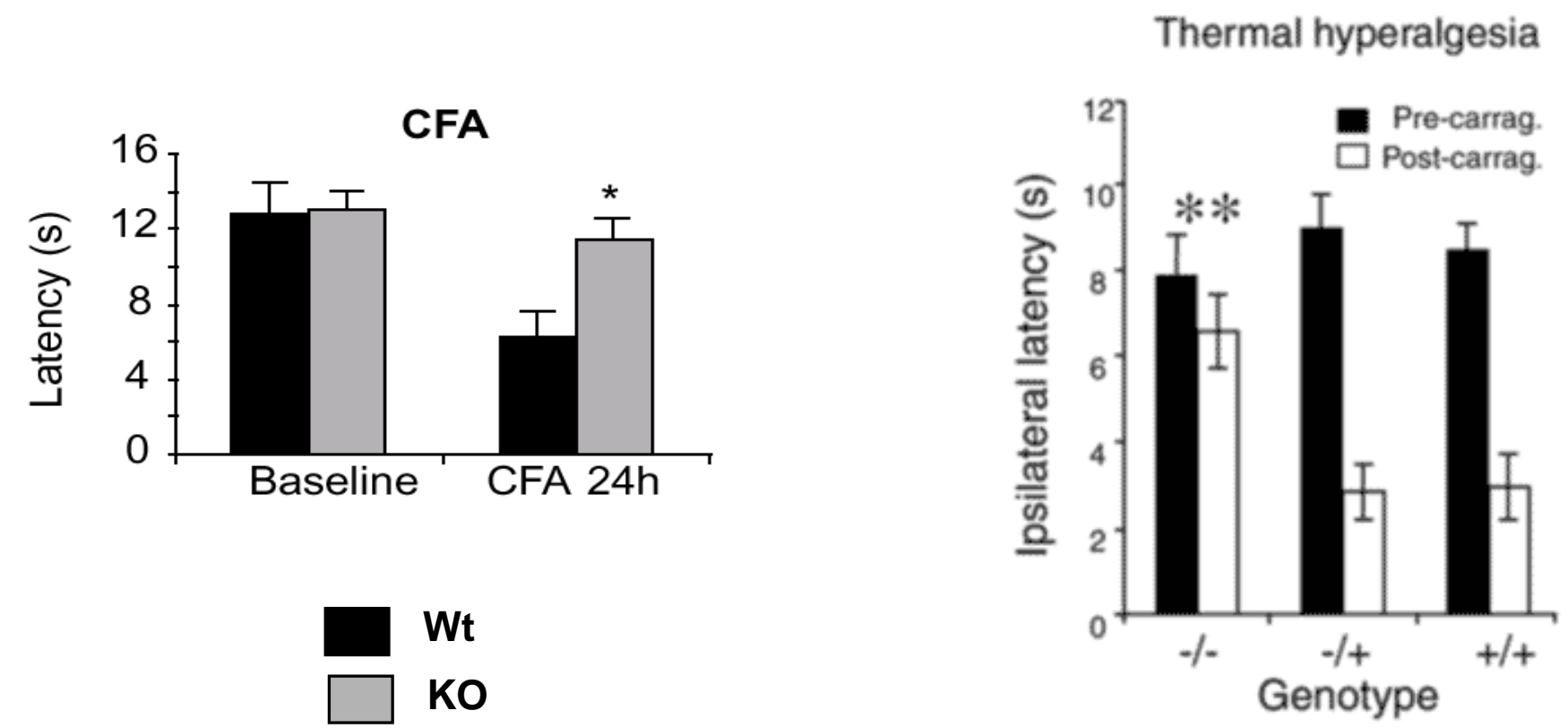

Davis et al (2000) Nature 405:183-7; Caterina et al (2000) Science, 288, 306-13

- $\quad$ TRPV1 expression in the spinal cord is up-regulated after SCI - Zhou et al (2002) J Surg Res. 107:140-4

- TRPV1 expression increased in inflamed human oesophagus - Mathews et al (2004) Eur J Gastroenterol Hepatol.16:897-902 


\section{Various distinct chemical scaffolds act as TRPV1 antagonists}

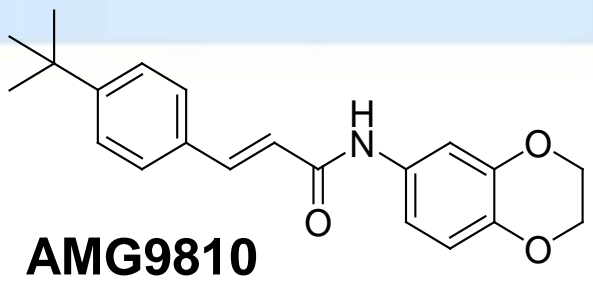

Gavva et al 2005 JPET, 313:474-484 $\mathrm{Cl}$<smiles>Cc1ccc(-c2cc3c(=O)[nH]c(=O)[nH]c3nc2C(C)(C)C)cc1</smiles>

NVS Pyridopyrimidine

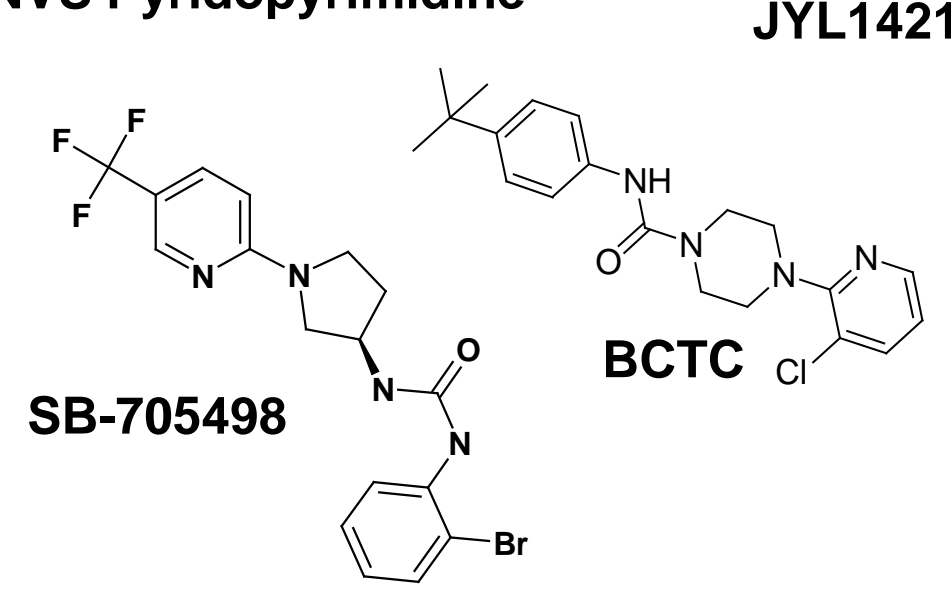

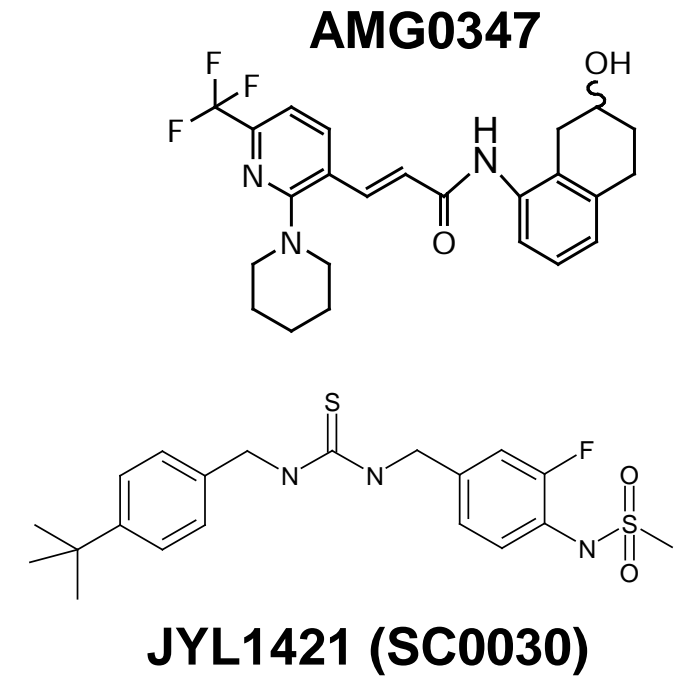
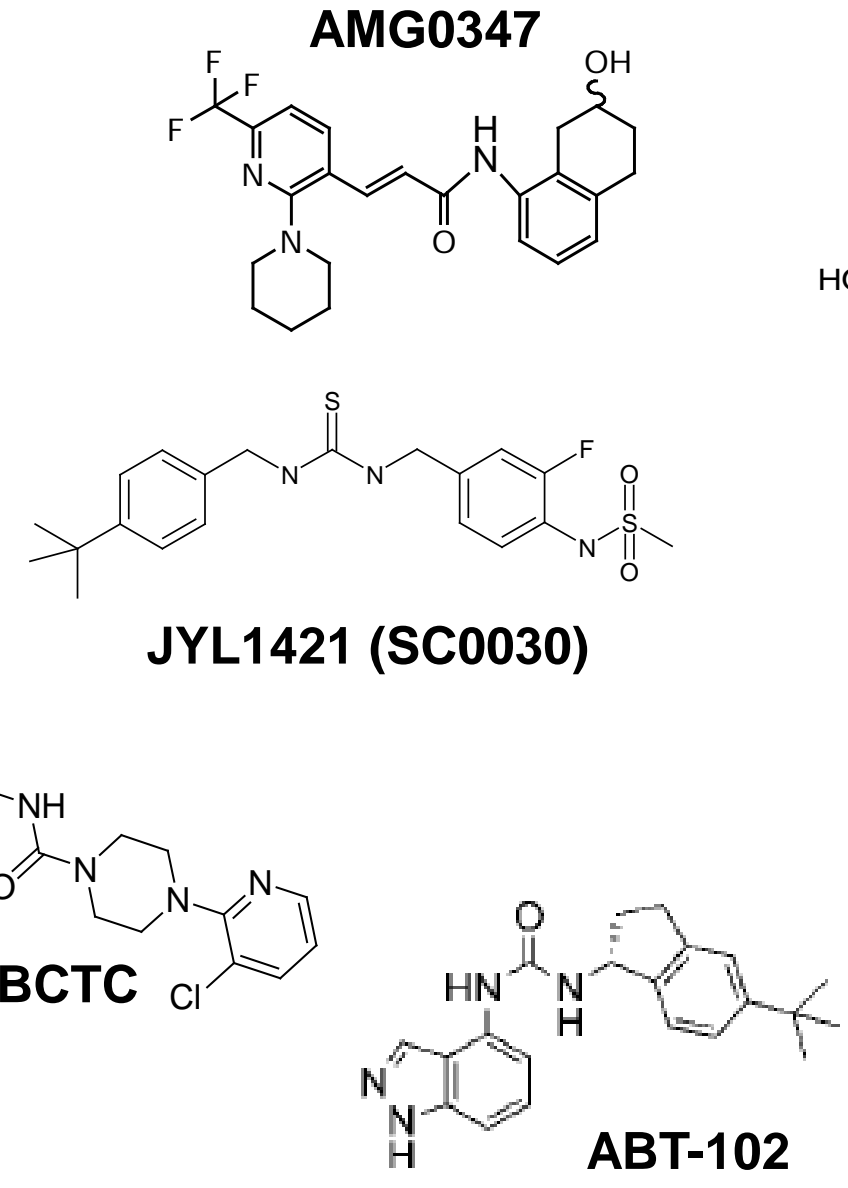
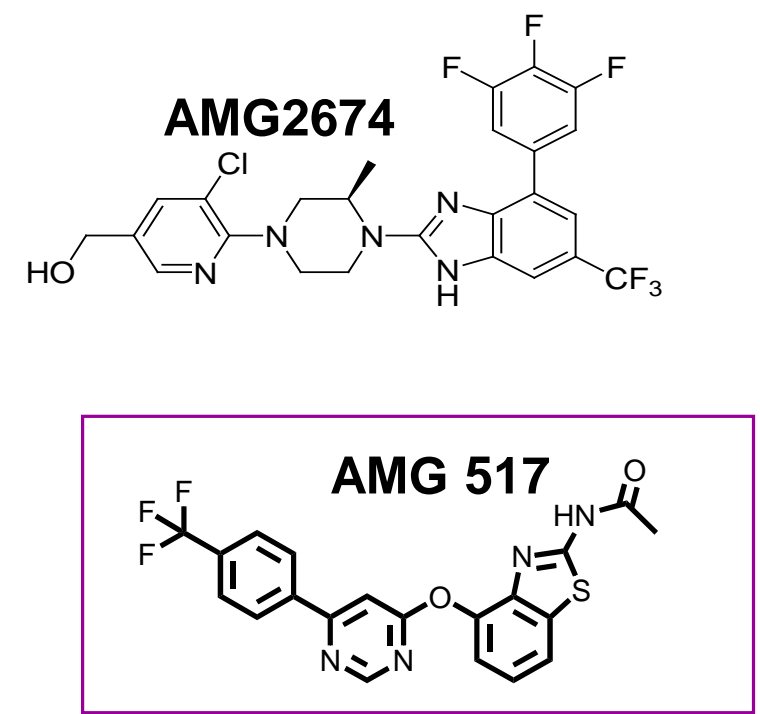

Doherty et al., 2007 JMC 50:3515-27 Gavva et al., 2007 JPET 323:128-137

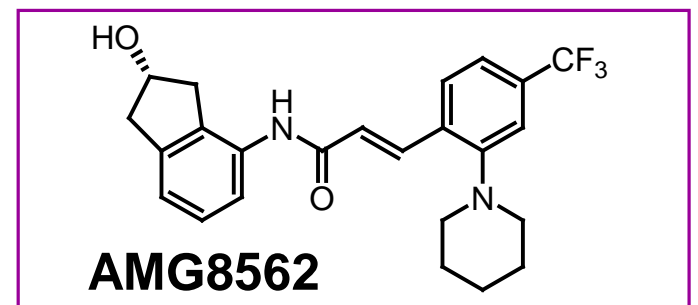




\section{Strategy for SAR development}

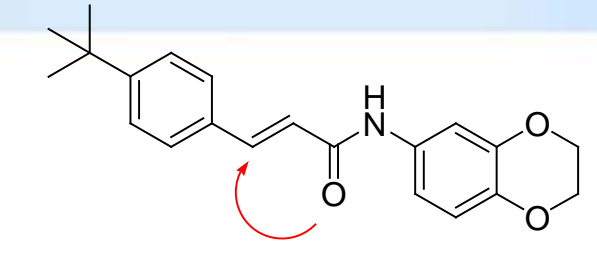

rTRPV1 (Cap) IC $\mathrm{C}_{50}=79 \mathrm{nM}$ rTRPV1 $(\mathrm{pH}) \quad \mathrm{IC}_{50}=350 \mathrm{nM}$

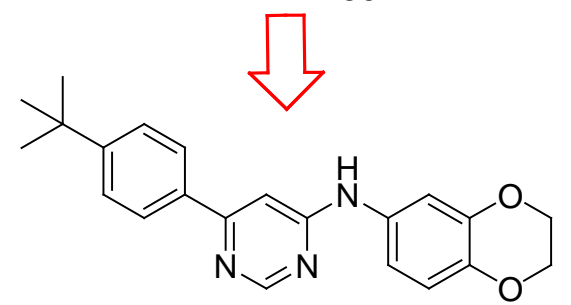

rTRPV1 (Cap) IC $\mathrm{I}_{50}=120 \mathrm{nM}$ rTRPV1 $(\mathrm{pH}) \quad \mathrm{IC}_{50}=680 \mathrm{nM}$

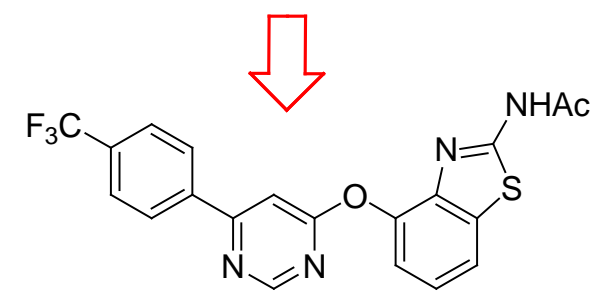

rTRPV1 (Cap) $I_{50}=0.9 \mathrm{nM}$ rTRPV1 $(\mathrm{pH}) \quad \mathrm{IC}_{50}=0.5 \mathrm{nM}$
- High-throughput screening of our corporate database identified several $N$-aryl cinnamides as TRPV1 antagonists

- Constrained analogs of S-cis cinnamides provided an entry into a novel chemical series of TRPV1 antagonists

- Further optimization lead to the clinical candidate AMG 517 


\section{AMG 517 blocks all modes of TRPV1 activation}

\section{Capsaicin activation}

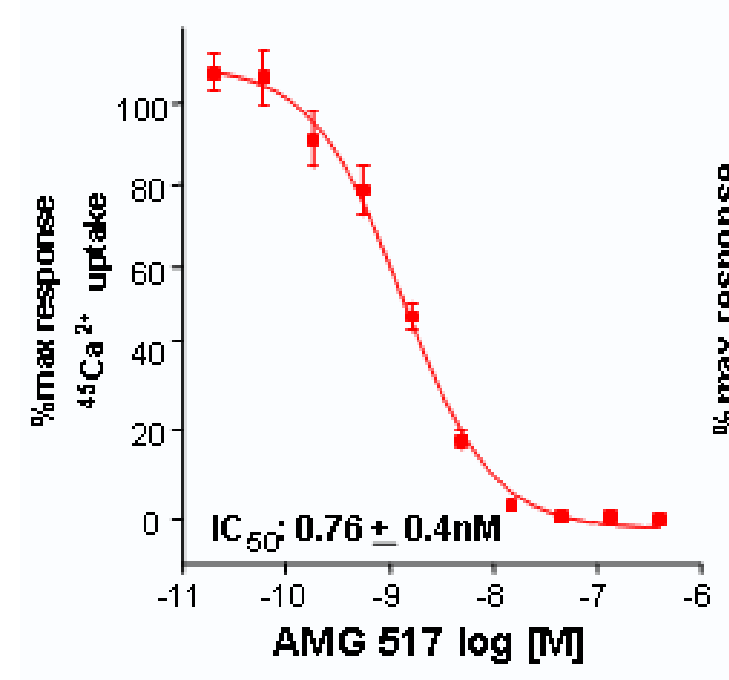

$\mathrm{pH} 5$ activation

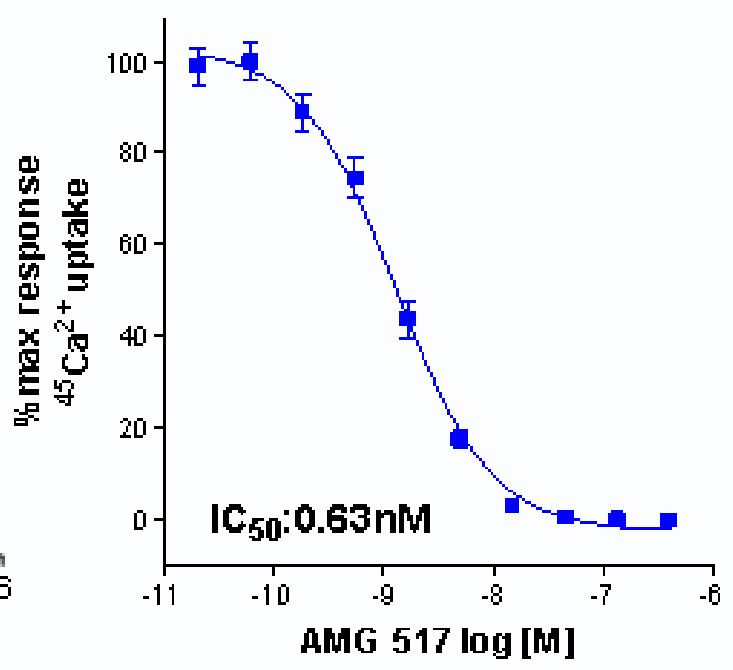

Heat $\left(45^{\circ} \mathrm{C}\right)$ activation

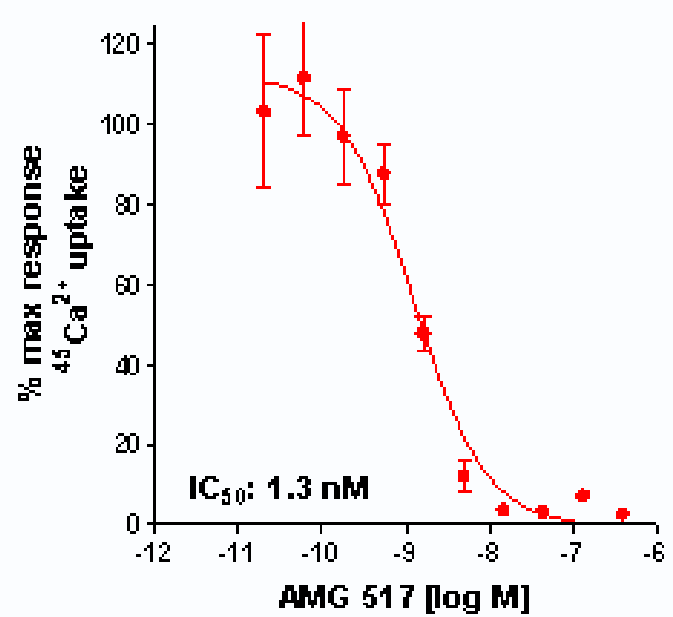

- $\quad$ AMG 517 is a potent antagonist of rodent, dog, monkey and human TRPV1

- $\quad$ AMG 517 does not block TRPA1, TRPV2, TRPV3, TRPV4, and TRPM8 


\section{AMG 517 blocks all modes of TRPV1 activation}

Capsaicin activation

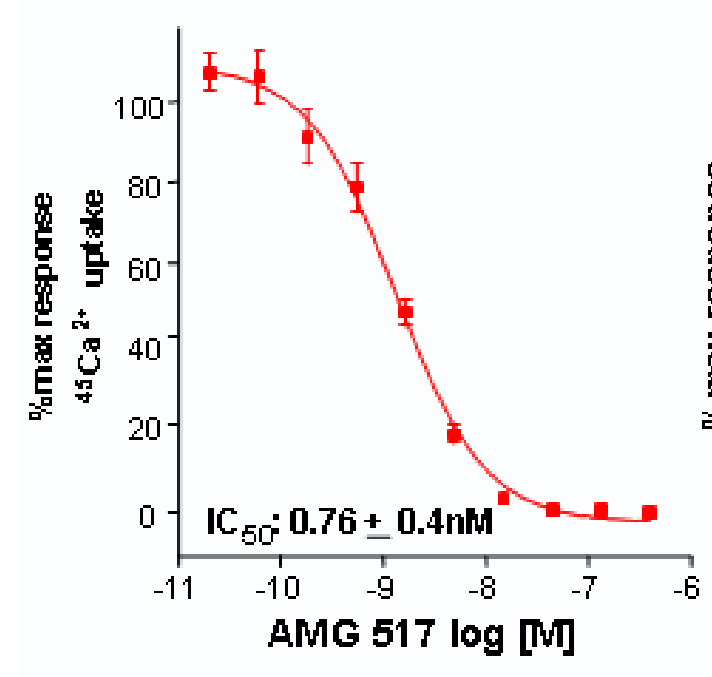

$\mathrm{pH} 5$ activation

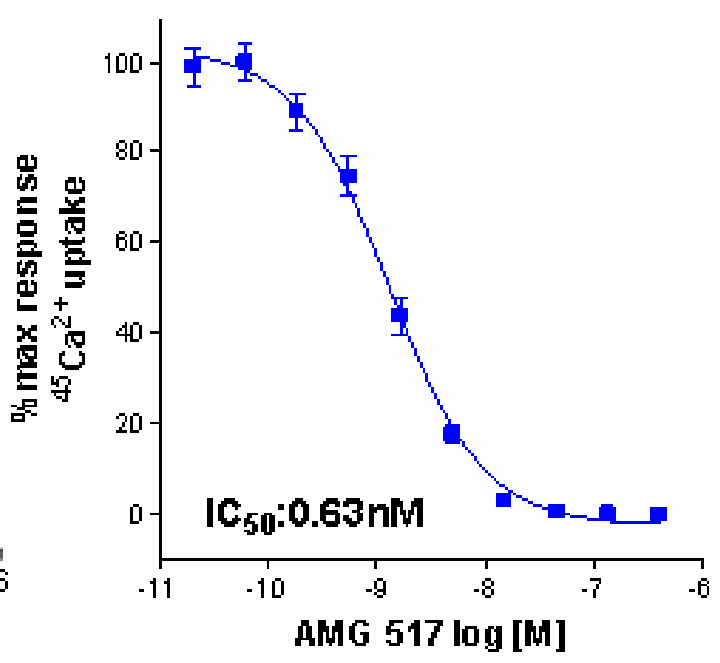

Heat $\left(45^{\circ} \mathrm{C}\right)$ activation

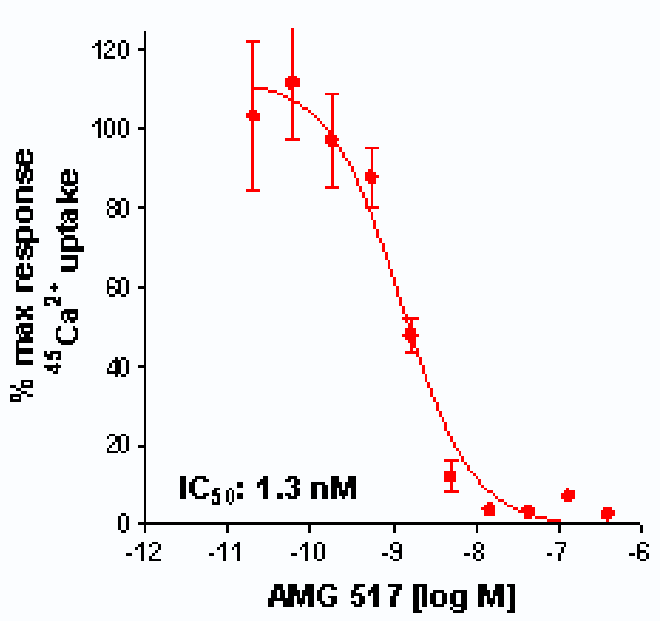

- $\quad$ AMG 517 is a potent antagonist of rodent, dog, monkey and human TRPV1

- $\quad$ AMG 517 does not block TRPA1, TRPV2, TRPV3, TRPV4, and TRPM8 


\section{AMG 517 blocks capsaicin-induced flinching in rats}

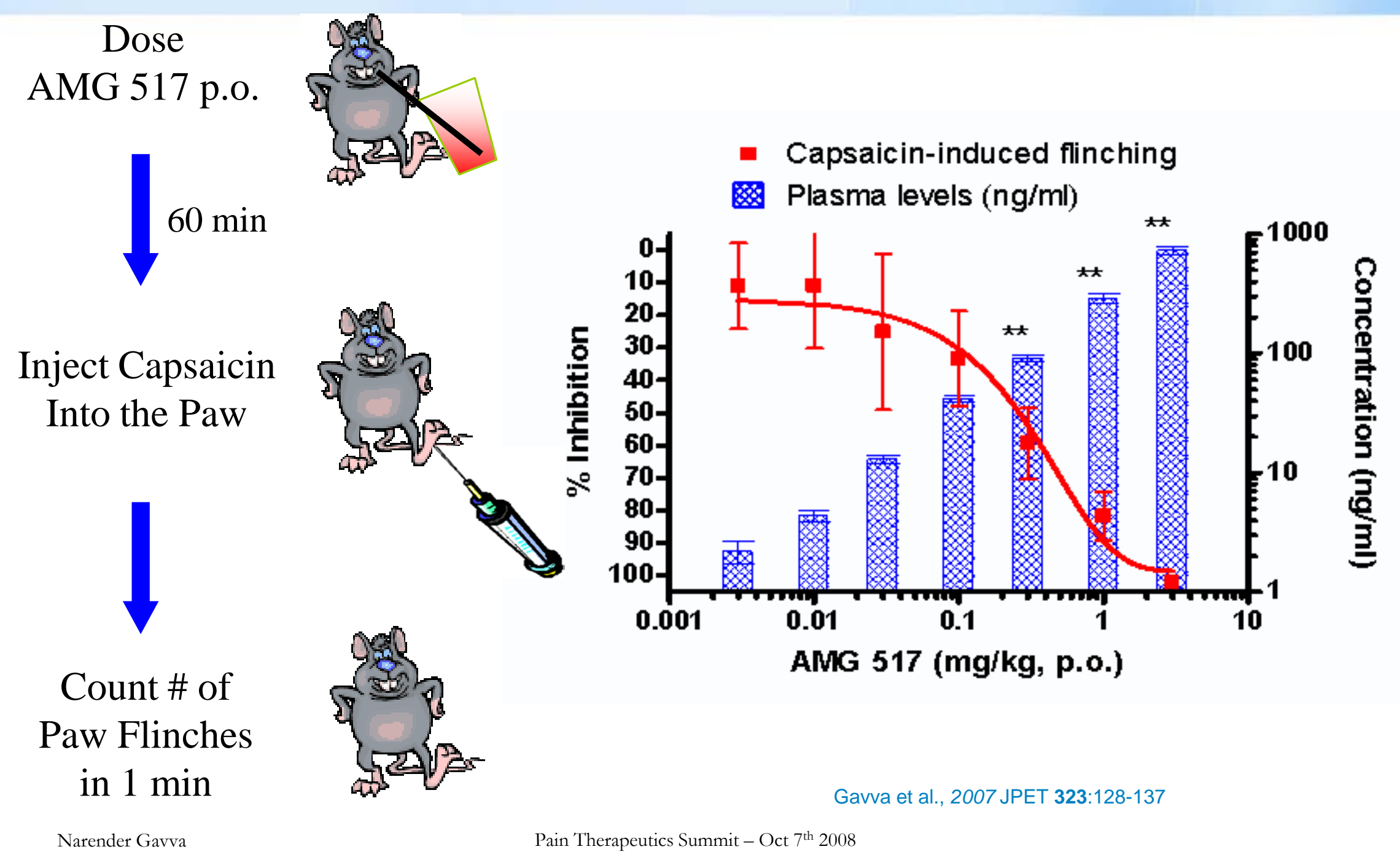




\section{AMG 517 blocks inflammation-induced thermal hyperalgesia}

Inject CFA

into the paw

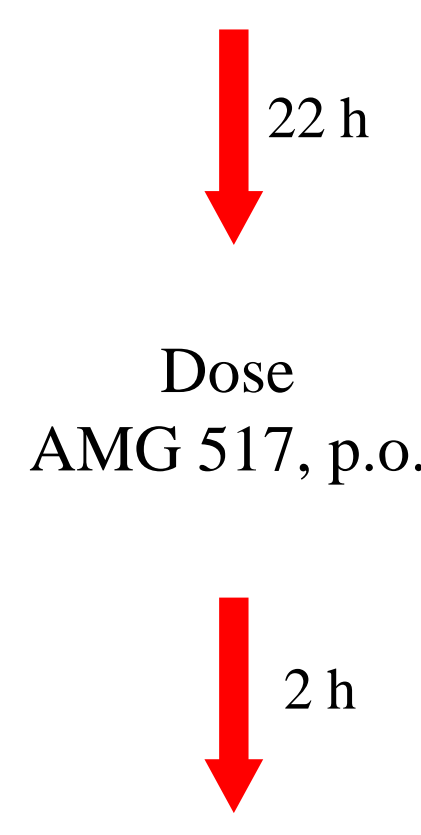

Record thermal sensitivity

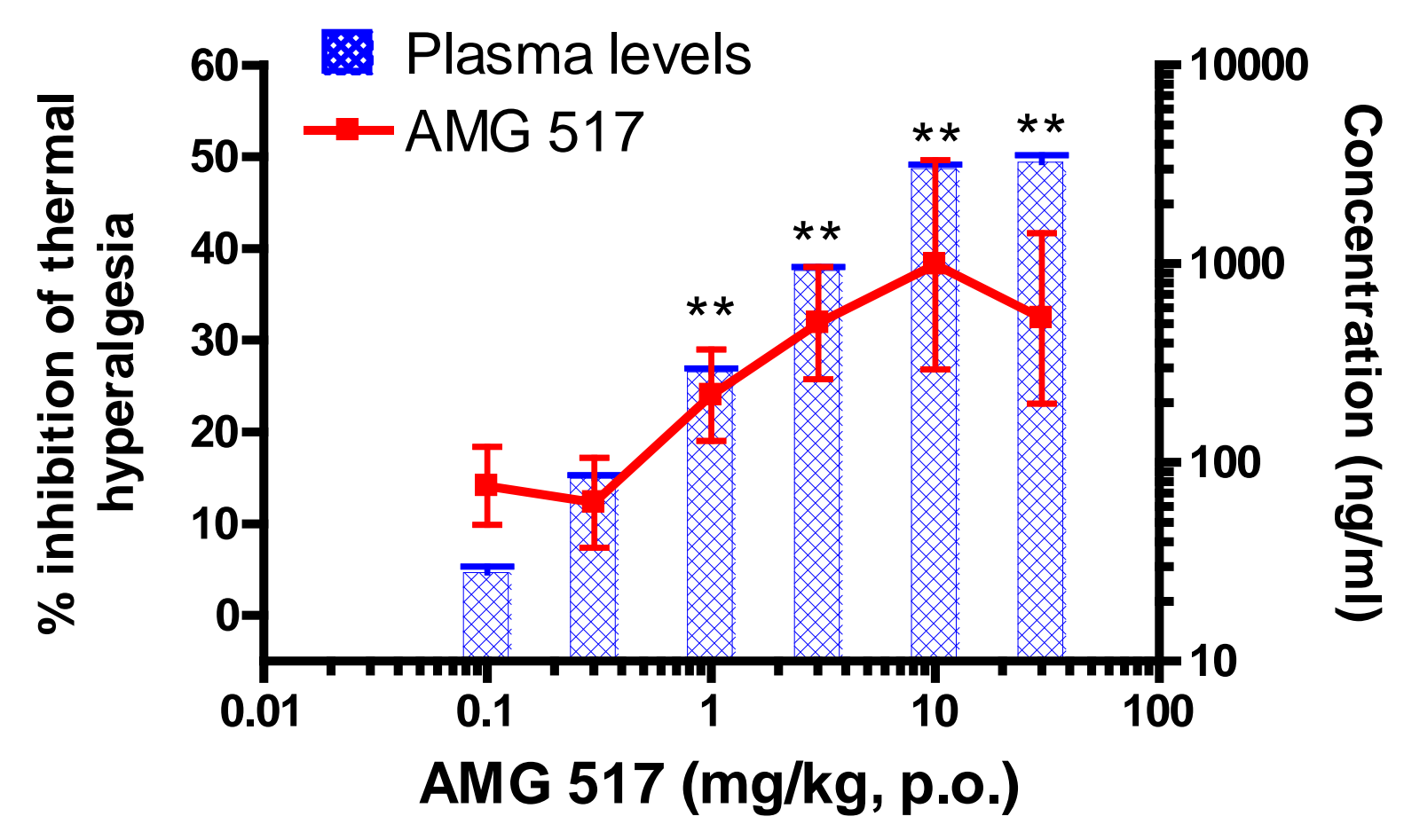

Gavva et al., 2007 JPET 323:128-137 


\section{Kґ V I antagonists DIOck capsa1cin-1nquced hypothermia and cause hyperthermia by themselves}

- $\quad$ On-target challenge model

- Capsaicin, a TRPV1 agonist produces an "on-target” decrease in body temperature

- $\quad$ Pretreatment with a TRPV1 antagonist should block capsaicin-induced hypothermia

$\rightarrow$ Vehicle/Vehicle $\rightarrow$ AMG9810/Vehicle

$\multimap-$ Vehicle/Capsaicin $\multimap$ AMG9810/Capsaicin
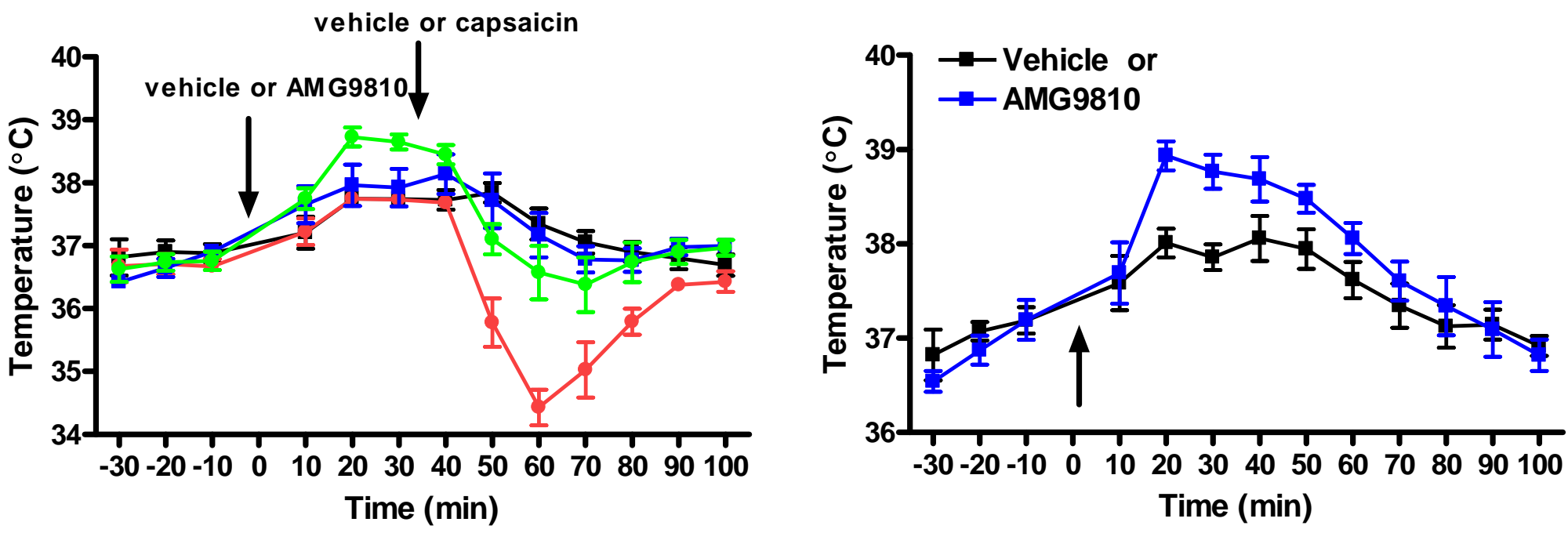

Gavva et al., J Neurosci. 2007 Mar 28;27(13):3366-3374 


\section{AMG 517 causes hyperthermia in multiple species}

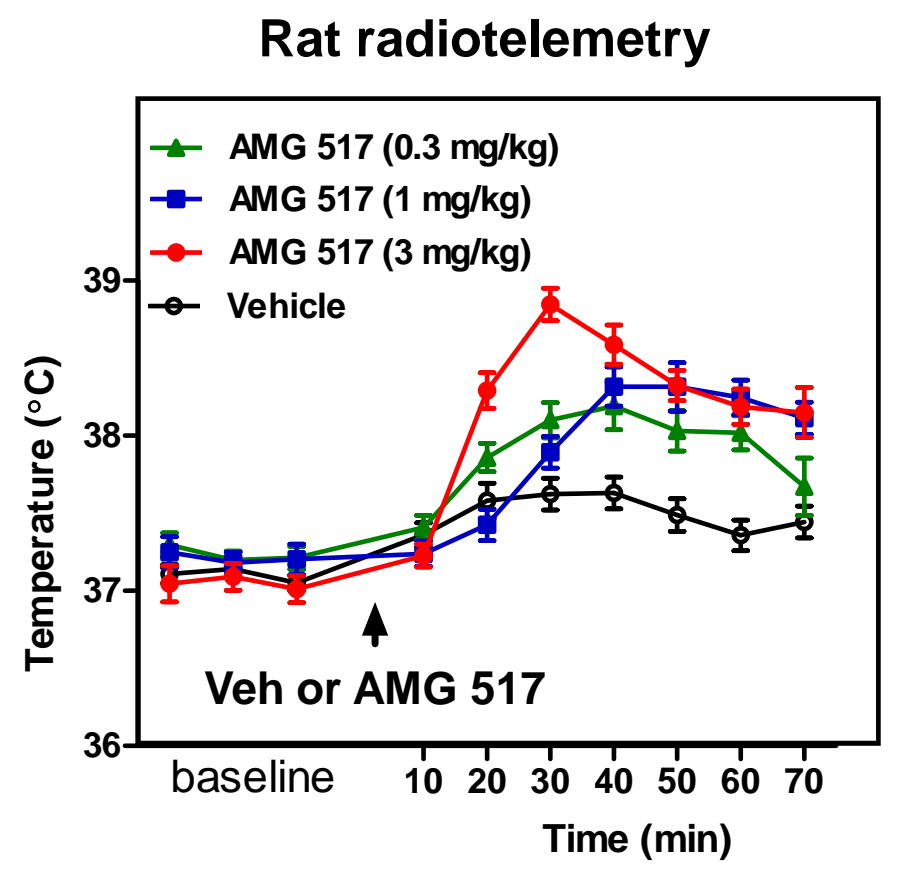

\section{Cynomolgus monkeys}

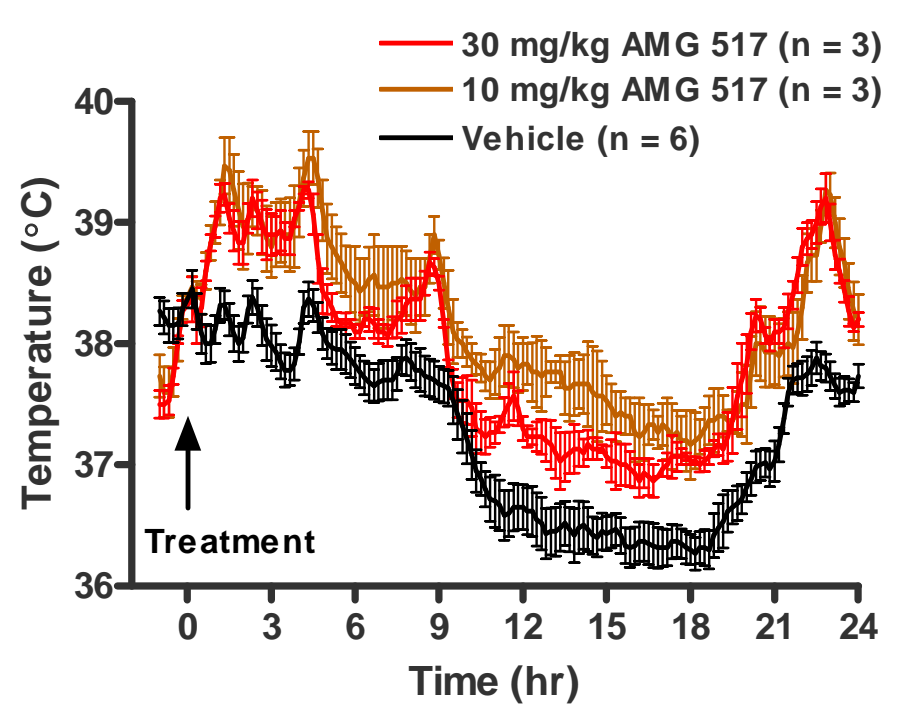

Gavva et al., JPET 2007 323:128-137

- $\quad$ TRPV1 antagonists caused hyperthermia in rodents, dogs, monkeys 


\section{Agonists and antagonists do not cause body temperature changes in TRPV1 knockout mice}

Agonist-induced hypothermia

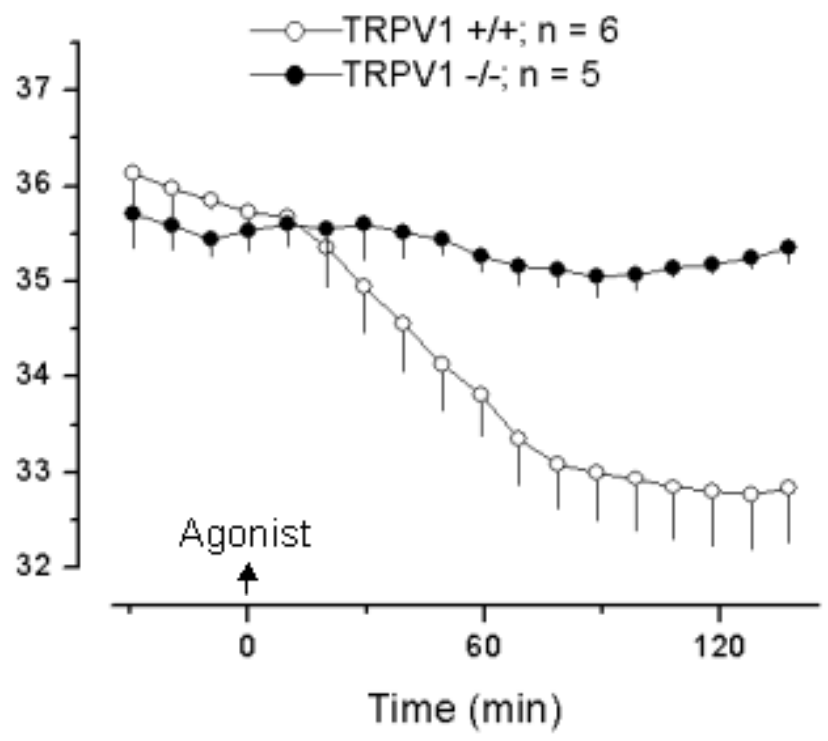

Antagonist-induced hyperthermia

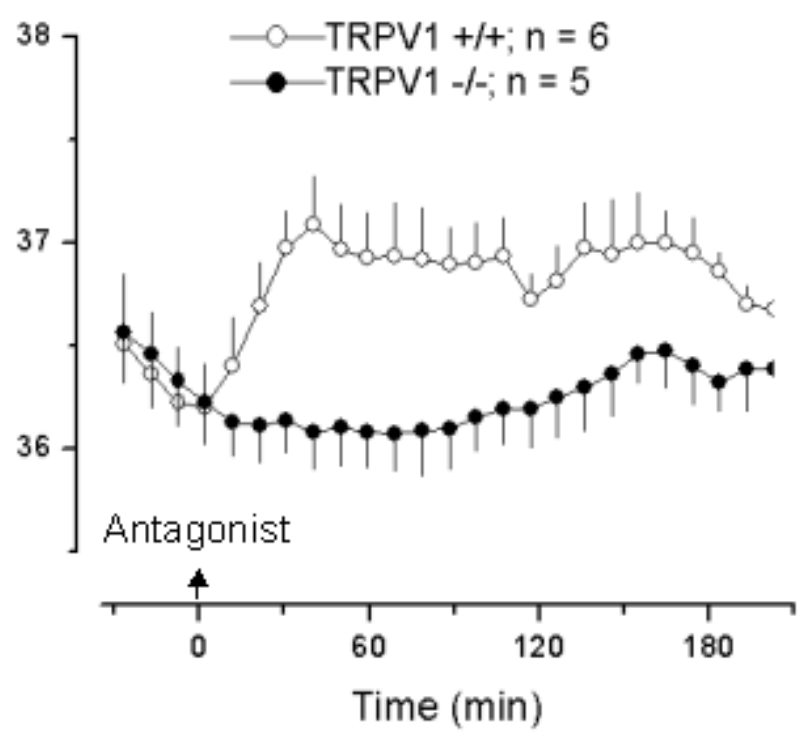

Steiner et al., J Neurosci. 2007 Jul 11;27(28):7459-68

- $\quad$ Agonist-induced hypothermia \& antagonist-induced hyperthermia are entirely TRPV1 mediated 


\section{AMG 517 causes hyperthermia by}

\section{vasoconstriction and increased thermogenesis}
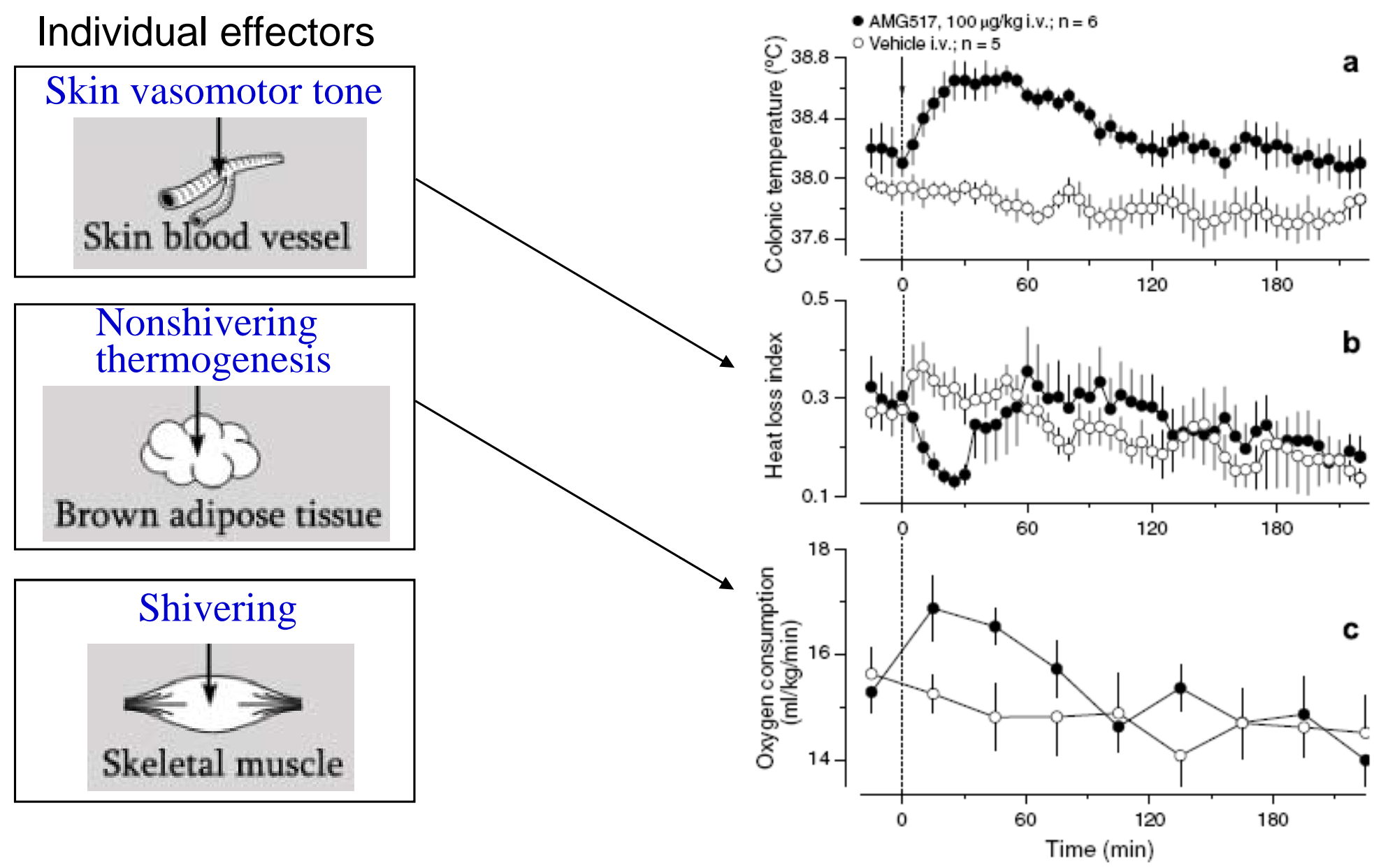

Gavva et al., 2008 Pain 136:202-210 


\section{Antipyretic acetaminophen suppresses TRPV1 antagonist-induced hyperthermia}

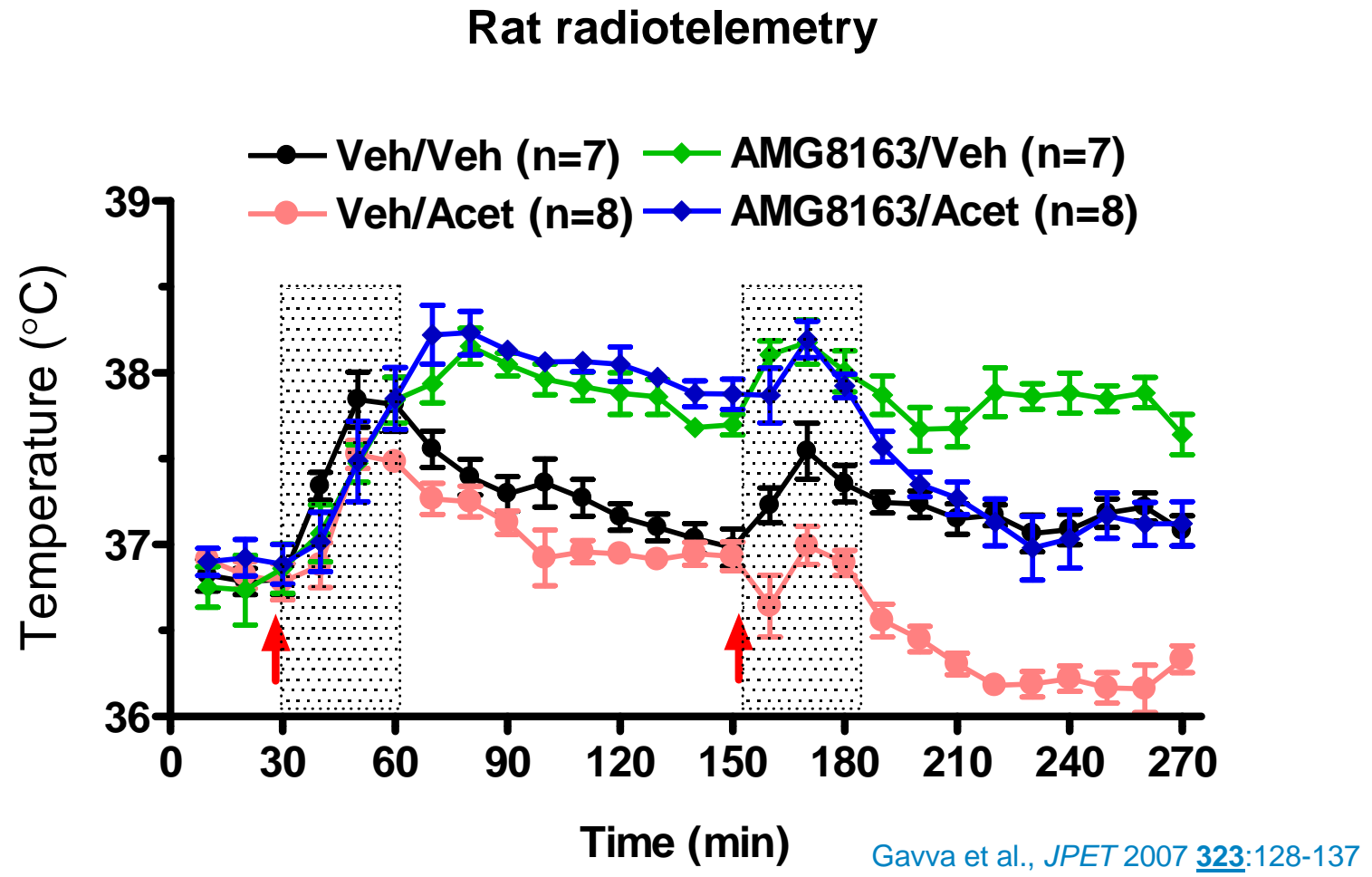

- AMG8163 is a 'boc' analogue of AMG 517

- Similar to AMG 517, AMG8163 blocks all modes of TRPV1 activation 


\section{Repeated administration of AMG 517 attenuates}

\section{hyperthermia}

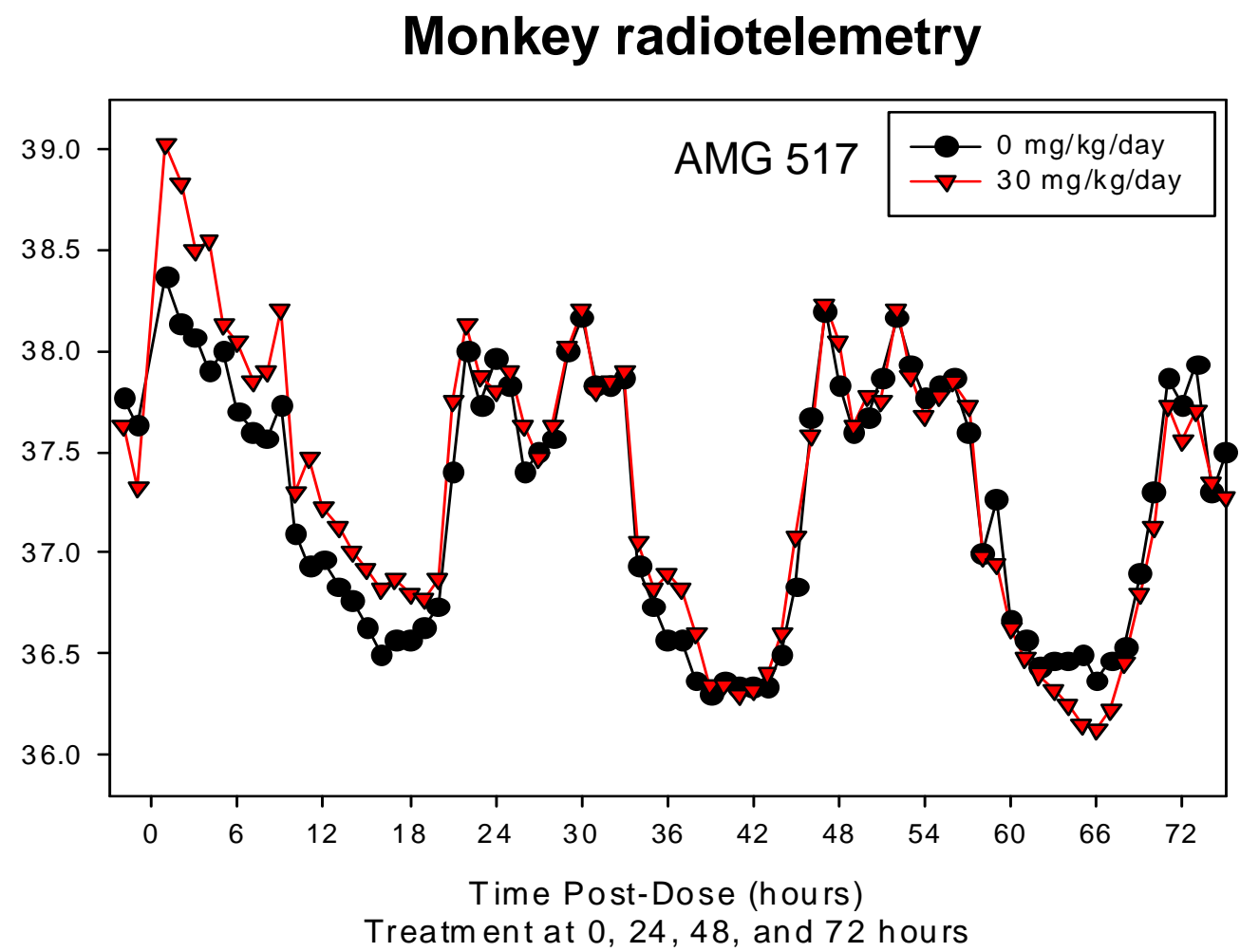

- $\quad$ TRPV1 is tonically activated and plays a role in body temperature regulation

- Role of TRPV1 in thermoregulation can be compensated 


\section{Clinical trial design}

- Vehicle of all studies: A suspension in a $100 \mathrm{ml}$ of $2 \%$ Pluronic 108 in OraPlus $尺$ followed by two $75 \mathrm{ml}$ water washes

- $1^{\text {st }}$ Phase I - single dose safety \& pharmacokinetic study

- Double-blind, placebo-controlled, randomized, single dose, dose-escalation sequential cohort study

- Healthy subjects received 1, 2, 5, 10, 20, or 25 mg AMG 517

- End points: number and incidence of treatment emergent-events, oral and tympanic body temperature measurements

- $2^{\text {nd }}$ Phase I - Repeated dose study

- Double-blind, placebo-controlled, randomized, single dose, dose-escalation sequential cohort study

- Placebo, 2, 5, or $10 \mathrm{mg}$ of AMG 517

- treatment emergent-events, difference in max body temperature on day 1 versus subsequent days, through day 7

- Phase lb - Dental pain study

- Double-blind, placebo-controlled, randomized, Parallel group, multi-center study

- Inclusion criteria: moderate to severe post-operative pain

- Single doses of placebo, 2, 8, or 15 mg of AMG 517 


\section{AMG 517 has a long half-life in healthy subjects}

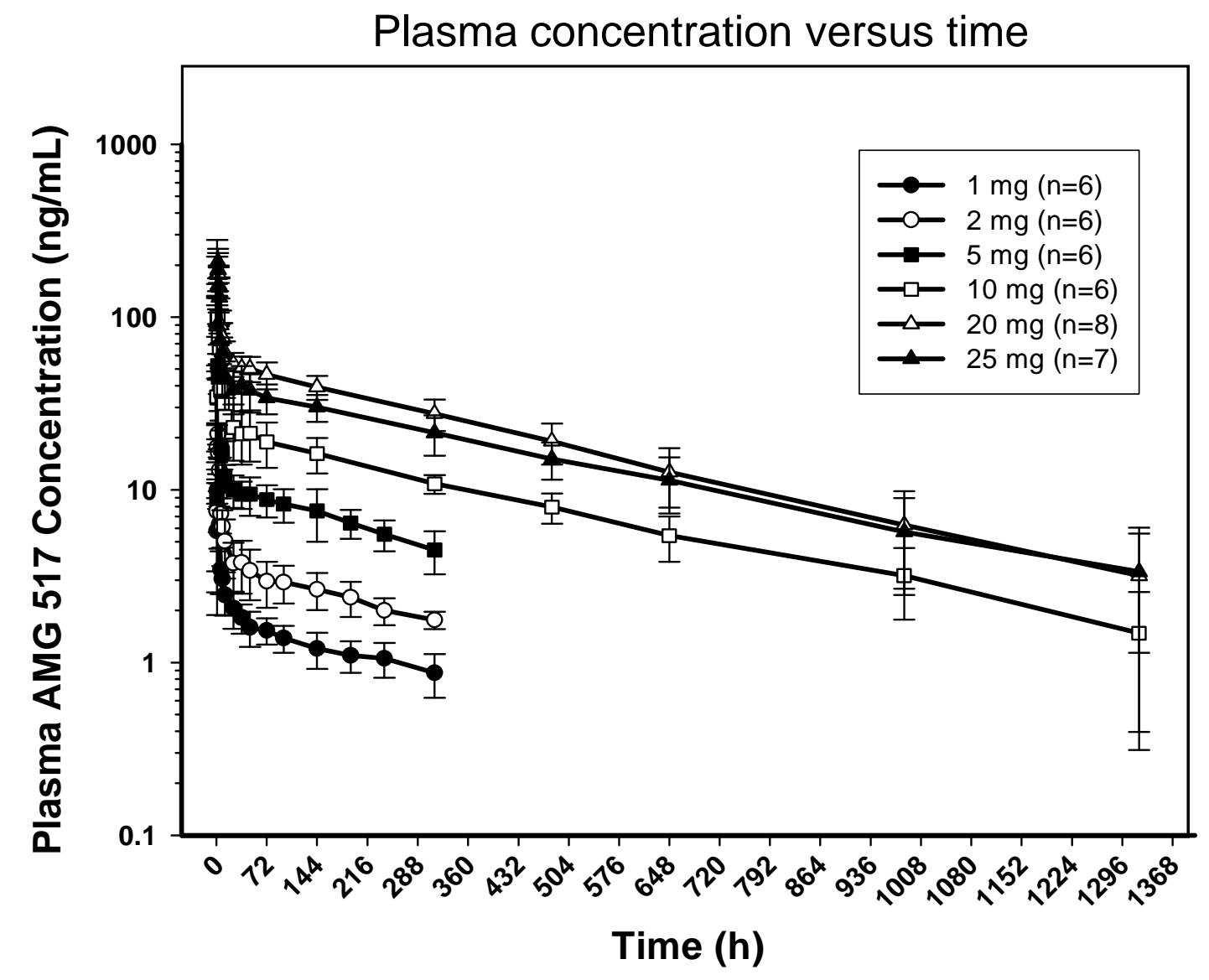

- Plasma half life: $\sim 300$ hrs 


\section{AMG 517 elicits transient hyperthermia in humans}

Body temperature \& Cmax versus time

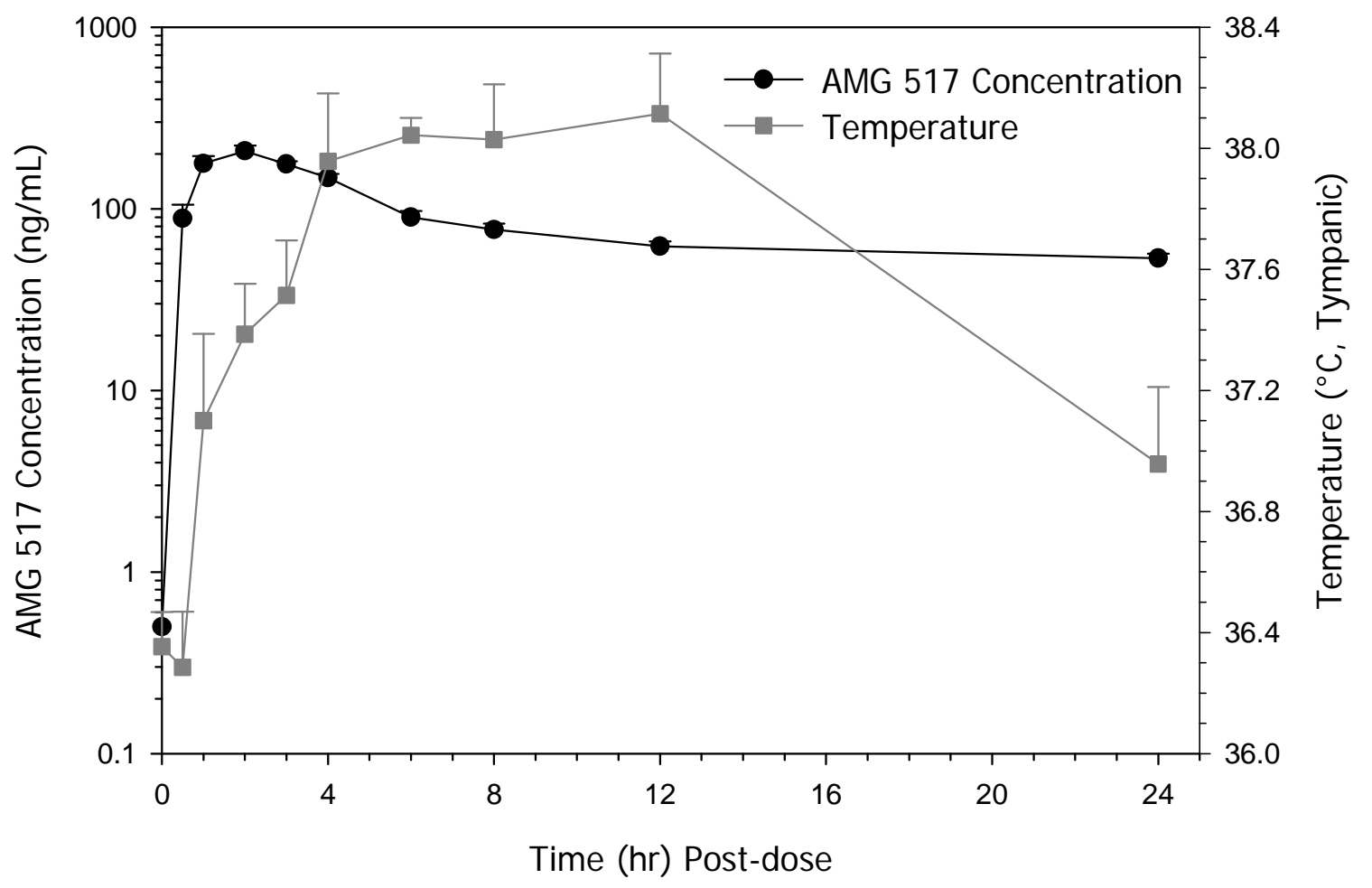

(mean \pm SD of the max body temperature [tympanic] presented) 


\section{AMG 517 caused plasma concentration- dependent hyperthermia in healthy subjects}

Body temperature versus $\mathrm{C}_{\max }$

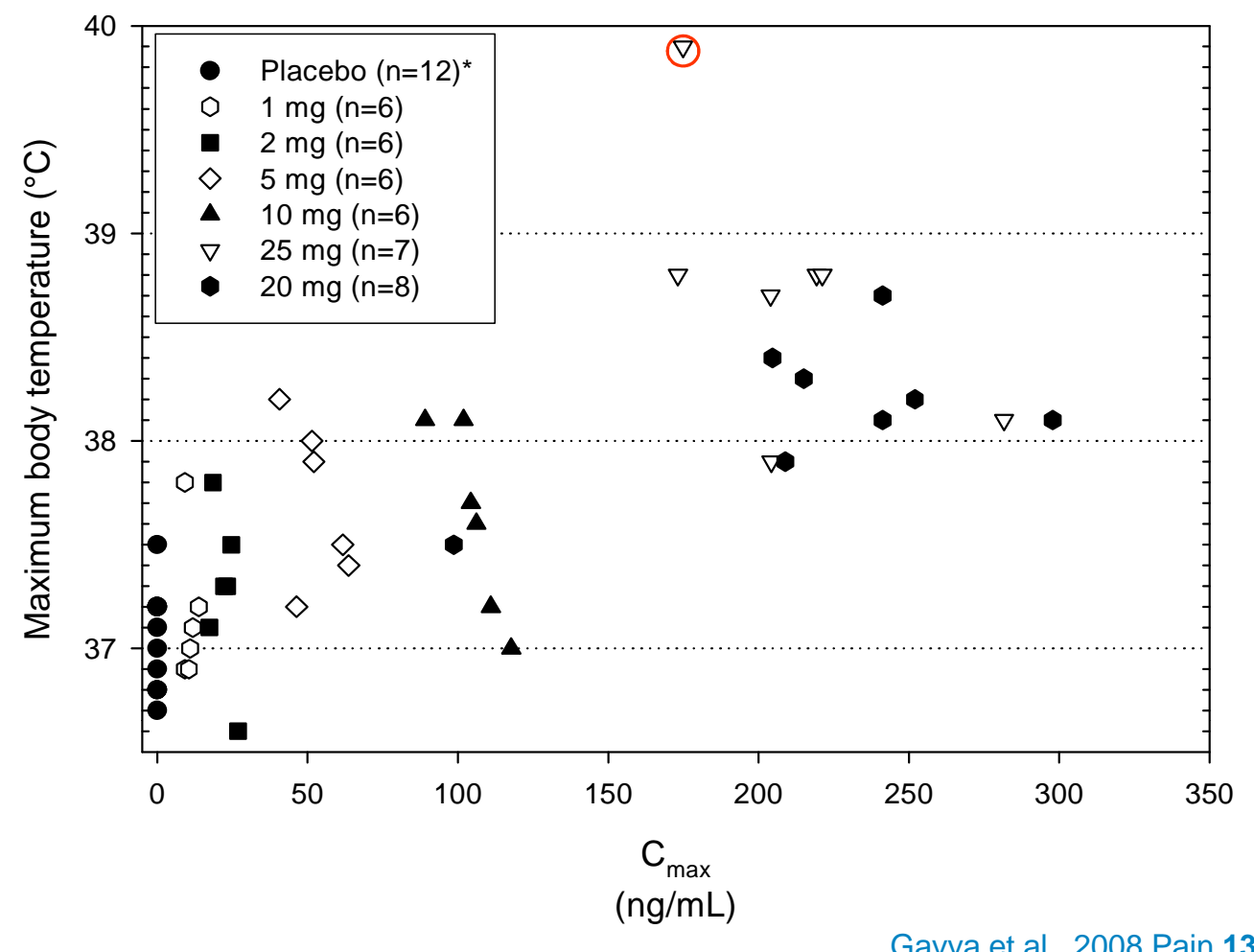

Gavva et al., 2008 Pain 136:202-210

- Single dose, oral suspension in a $100 \mathrm{~mL}$ of $2 \%$ Pluronic 108 in OraPlus ${ }^{\circledR}$

- $\quad$ Plasma half life of AMG 517 in humans: $\sim 300$ hrs 


\section{Trend of hyperthermia attenuation after repeated administration of AMG 517}

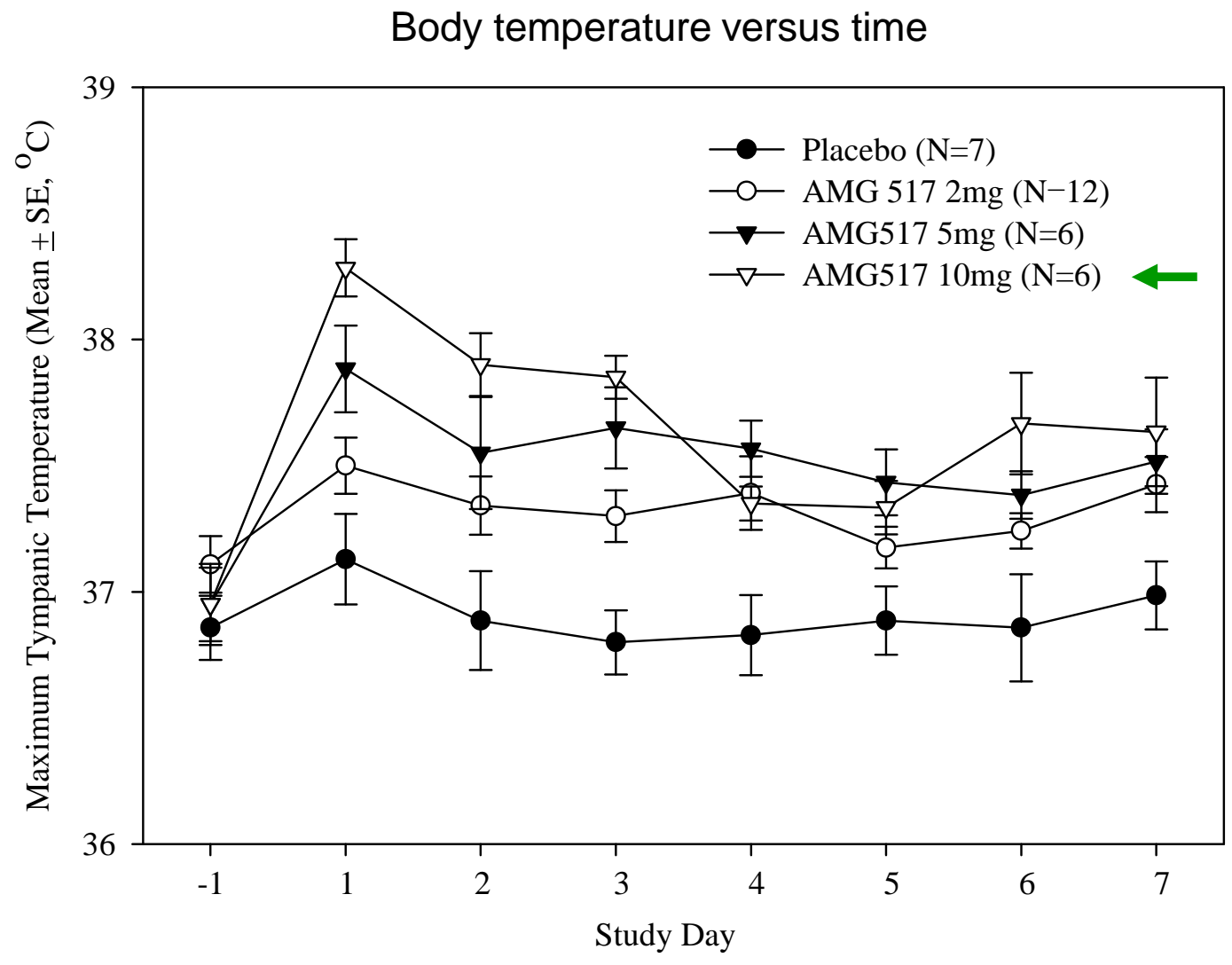

(mean \pm SD of the max body temperature [tympanic] presented) 


\section{AMG 517 caused marked hyperthermia in subjects who underwent molar extraction}

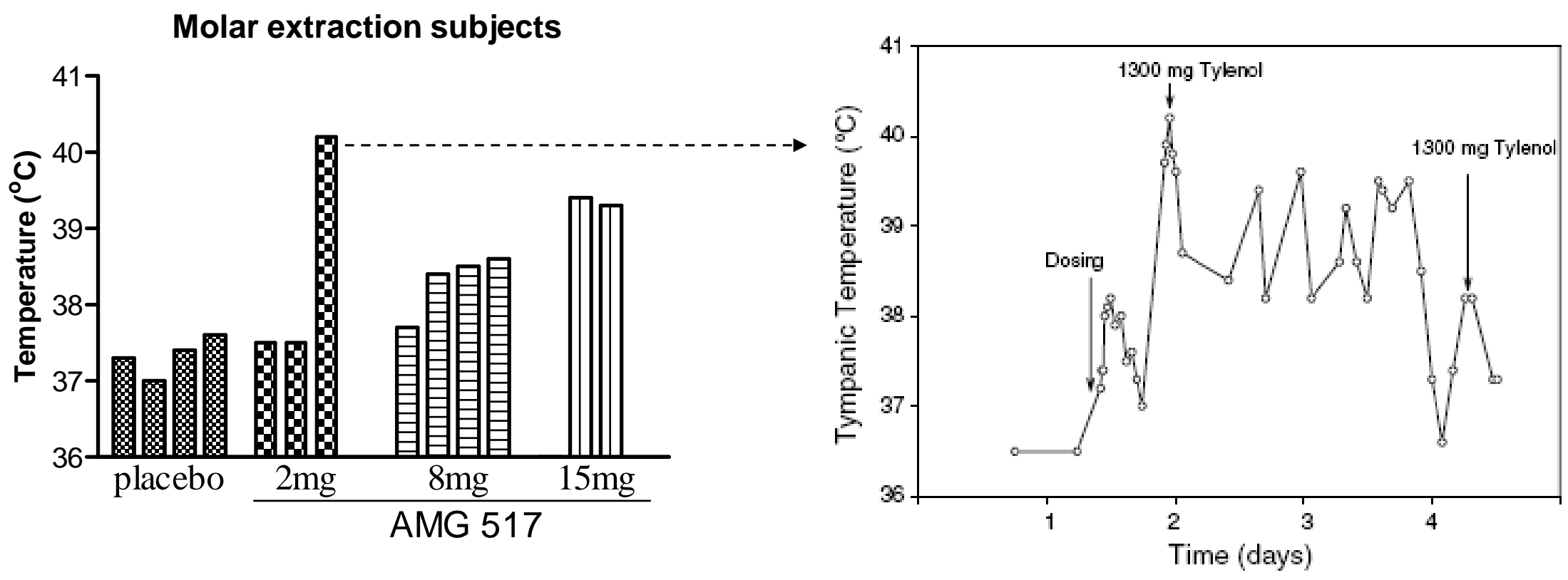

- $\quad$ TRPV1 antagonists causing hyperthermia in rodents, dogs, monkeys, and humans indicates an evolutionarily conserved role of TRPV1 in thermoregulation 


\section{First approach to address hyperthermia: Peripheral restriction of antagonists}

\section{Hypothesis}

- Thermoregulation is CNS mediated

- Can we minimize hyperthermia by restricting antagonists to the periphery?

\section{Strategy}

- Reduce brain exposure: peripherally restricted TRPV1 antagonists

- Increase polar surface area (PSA)

- Decrease logP

- Increase number of hydrogen-bond donors 


\section{Profiles of peripherally restricted TRPV1 antagonists}

\begin{tabular}{|l|c|c|c|c|}
\hline Compound & PSA & clogP & Cap IC $_{50}$ & B/P ratio \\
\hline AMG13731 & 145 & 3.9 & 2.9 & 0.02 \\
\hline AMG21629 & 128 & 3.5 & 0.5 & 0.05 \\
\hline AMG32915 & 141 & 1.9 & 0.2 & 0.04 \\
\hline AMG41394 & 132 & 3.9 & 1.3 & 0.04 \\
\hline
\end{tabular}




\section{Effect of AMG13731 and AMG21629 on rat body core temperature}
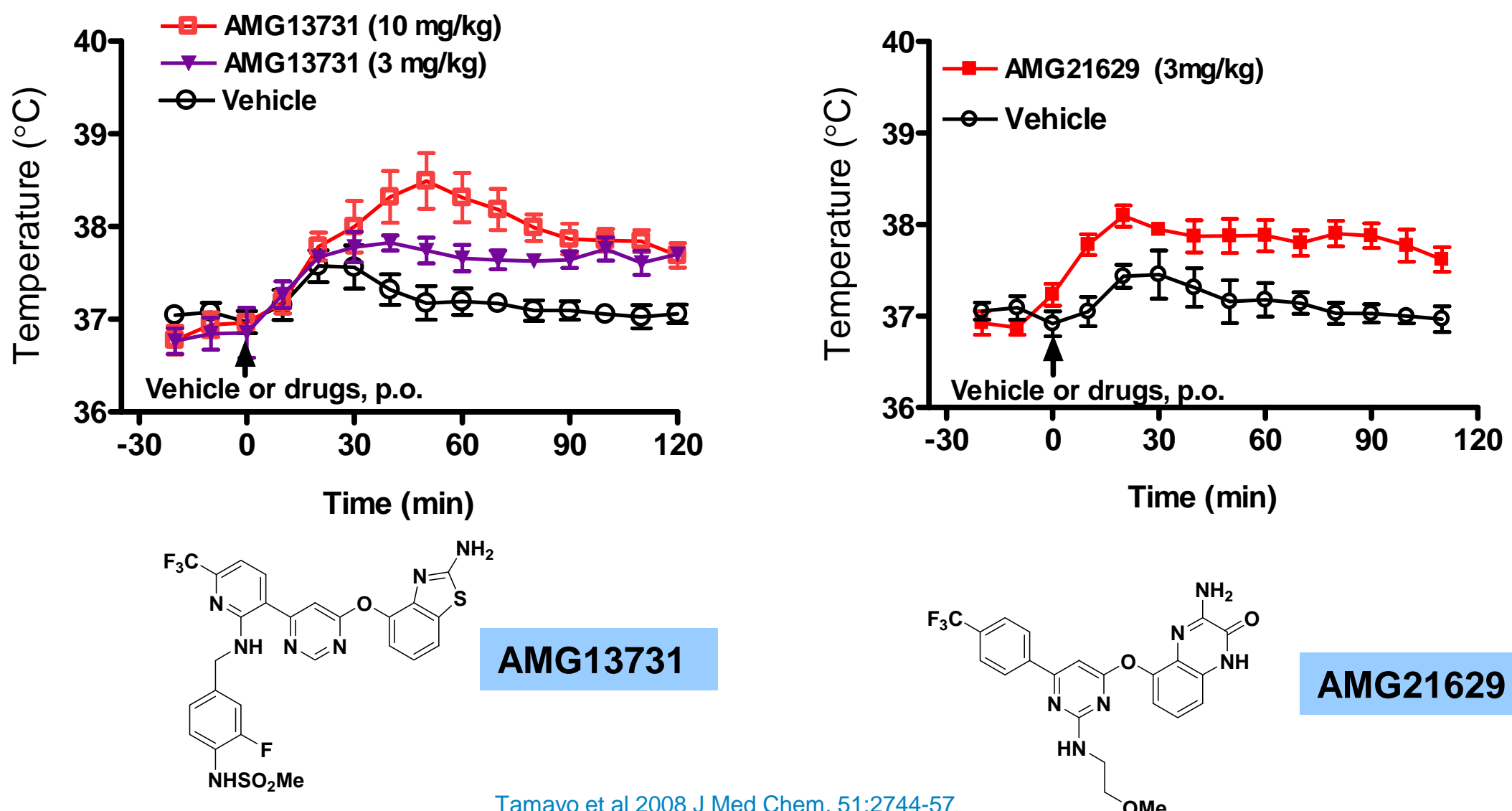

AMG13731

Tamayo et al 2008 J Med Chem. 51:2744-57

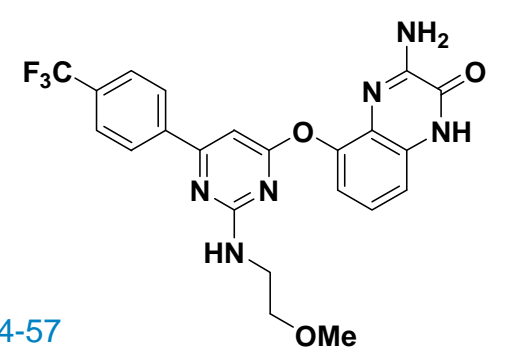

AMG21629

- $\quad$ Site of action for TRPV1 antagonist-induced hyperthermia is outside the BBB 


\section{Evaluation of compounds exhibiting differential pharmacology - $2^{\text {nd }}$ Approach}

\begin{tabular}{l|c|c|c|c|}
\cline { 2 - 5 } & Capsaicin & pH 5 & Heat & $\begin{array}{c}\text { Body } \\
\text { temp }\end{array}$ \\
\cline { 2 - 5 } Profile A & Block & Block & Block & $\checkmark$ \\
\cline { 2 - 5 } Profile B & Block & Partial block & Block & $\checkmark$ \\
\cline { 2 - 5 } Profile C & Block & Potentiate & No effect & $-\downarrow$ \\
\cline { 2 - 5 } Profile D & Block & Potentiate & Potentiate & \\
\cline { 2 - 5 } & & &
\end{tabular}

- Profiles defined by agonist-induced ${ }^{45} \mathrm{Ca}^{2+}$ uptake assays 


\section{AMG8562, a profile $C$ compound that does not cause hyperthermia in rats}
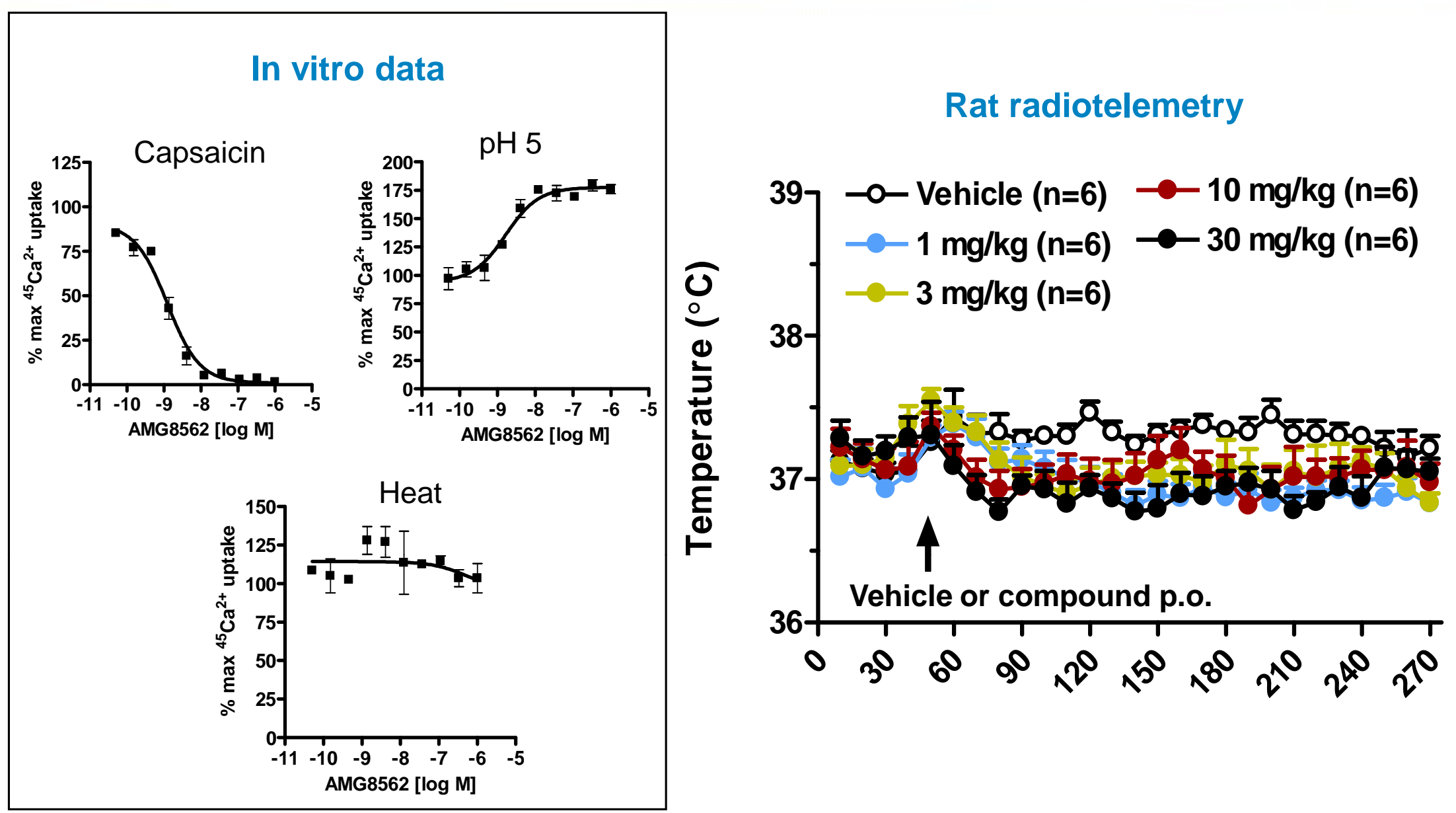

Lehto et al 2008 JPET 326:218-229 


\section{AMG8562 is modestly effective in capsaicin-challenge and pain models}

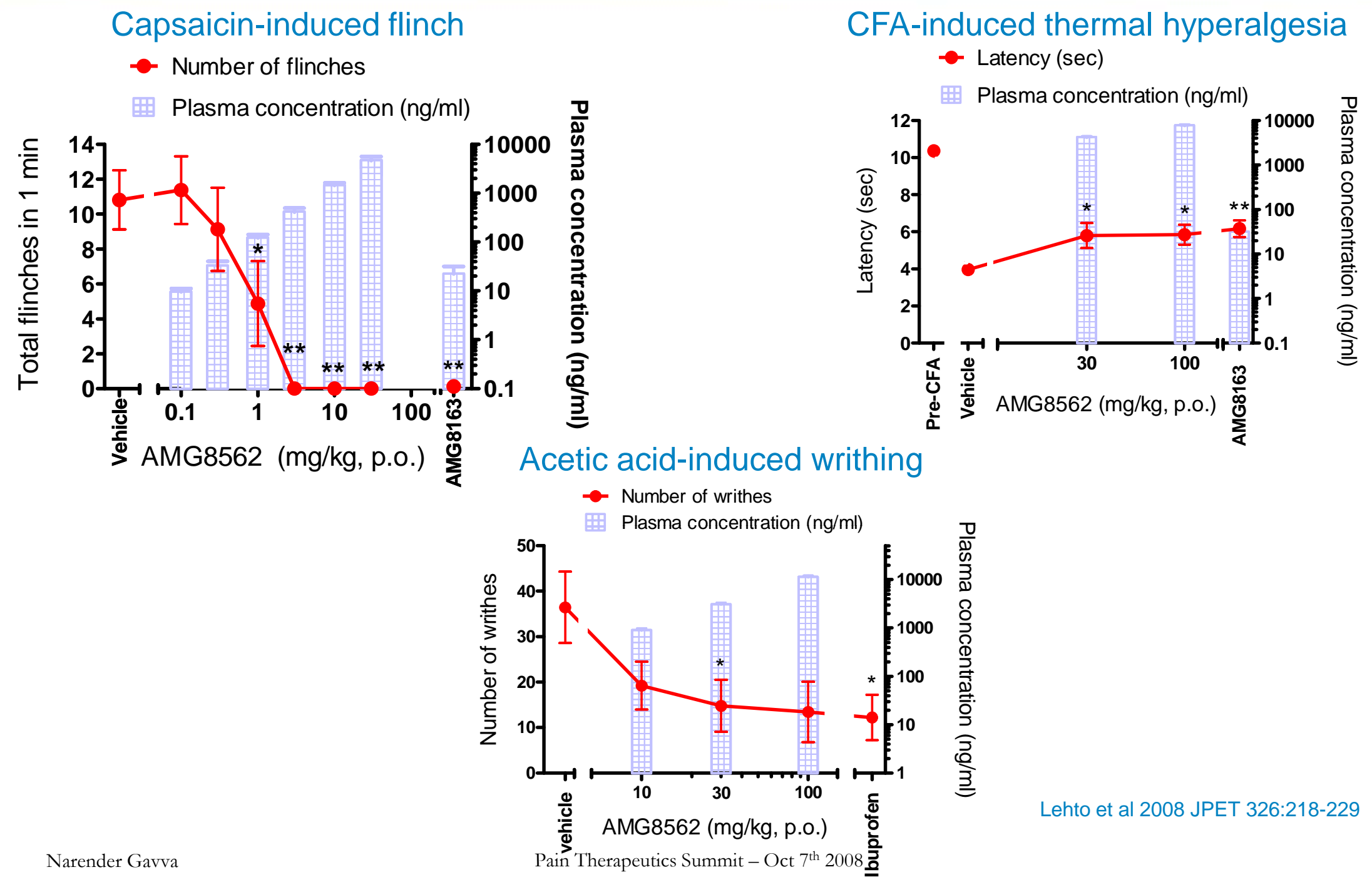




\section{AMG8562, exhibits profile B modulation of human TRPV1}

Capsaicin activation

pH 5 activation

Heat activation
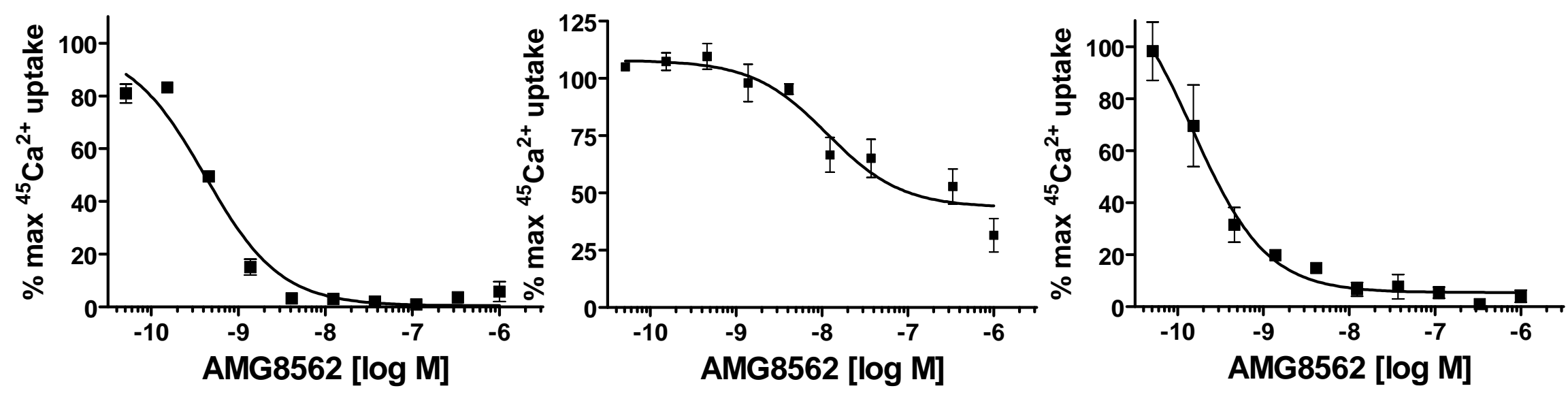

Lehto et al 2008 JPET 326:218-229

- $\quad$ Profile B modulators cause hyperthermia in rats 


\section{Current status of TRPV1 antagonists in the clinic}

\begin{tabular}{|l|l|l|l|l|l|}
\hline $\begin{array}{l}\text { Compound } \\
\text { name }\end{array}$ & Company & $\begin{array}{l}\text { Route of } \\
\text { administration }\end{array}$ & Indication & Stage & $\begin{array}{l}\text { Current } \\
\text { status }\end{array}$ \\
\hline ABT-102 & Abbott & Oral & & Phase I & Initiated ? \\
\hline AMG 517 & Amgen & Oral & Dental pain & Phase Ib & Terminated \\
\hline AZD1386 & AstraZeneca & Oral & Dental pain & Phase II & Completed \\
\hline GRC 6211 & Lilly/Glenmark & Oral & Dental pain & Phase II & Suspended \\
\hline JTS-653 & Japan Tobacco & Oral & $\begin{array}{l}\text { Overactive } \\
\text { bladder } \\
\text { pain }\end{array}$ & Phase I & On going \\
\hline MK 2295 & Merck/Neurogen & Oral & Dental pain & Phase II & Completed \\
\hline SB-705498 & GSK & Oral & Migraine & Phase II & Terminated \\
\cline { 4 - 6 } & & & Rectal pain & Phase II & Terminated \\
\cline { 4 - 6 } & & & Dental pain & Phase II & Completed \\
\hline PF-4065463 & Evotec/Pfizer & unknown & unknown & unknown & unknown \\
\hline
\end{tabular}




\section{Conclusions}

- $\quad$ Genetic and pharmacological evidence suggests that TRPV1 contributes to hyperalgesia

- $\quad$ TRPV1 antagonists block capsaicin-induced flinch (on-target challenge) and act as anti-hyperalgesics

- $\quad$ AMG 517, a selective TRPV1 antagonist caused plasma concentration dependent hyperthermia in humans

- Antagonist-induced hyperthermia showed a complete and clear attenuation after repeated dosing in preclinical species but not in humans

- Mechanisms of hyperthermia include antagonist-induced vasoconstriction and increased thermogenesis

- $\quad$ Peripherally restricted TRPV1 antagonists caused hyperthermia

- $\quad$ Profile C modulators of TRPV1 did not cause hyperthermia and demonstrated modest efficacy in pain models

- Clinical trial results of other TRPV1 antagonists are eagerly awaited 


\section{Outstanding questions and next steps}

\section{Preclinical}

- $\quad$ 'Profile C' modulators of both rodent and human TRPV1

- $\quad$ Efficacy after repeated dosing of TRPV1 antagonists

- $\quad$ TRPV1 function in non-pathophysiological states (e.g., thermosensation)

Clinical

- Development by different routes of administration (e.g., topical application)

- Development of short half-life antagonists

- Development of antipyretic + TRPV1 antagonist combination

- Development of TRPV1 antagonists in primary indications (e.g., Osteoarthritis) 


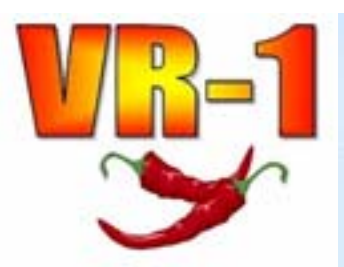

\section{The Team}

\begin{tabular}{ll} 
Departments & Neuroscience \\
\cline { 2 - 2 } Neuroscience & Leyla Arik \\
PKDM & Anthony Bannon \\
Modeling & James Davis \\
E-phys & Narender Gavva \\
Sample Bank & Charles Henley \\
Regulatory Affairs & Gal Hever \\
Toxicology & Rongzhen Kuang \\
Pharmaceutics & David Immke \\
Clinical & Sonya Lehto \\
Analytical & Lana Klionsky \\
Legal & Ella Magal \\
Project Management & Licheng Shi \\
Marketing & Rami Tamir \\
& Jue Wang \\
& Judy Wang \\
& Weiya Wang \\
& Gary Zajic \\
& Dawn Zhu
\end{tabular}

Chemistry
Yunxin Bo
Ning Chen
Balan Chenera
Darin D'Amico
Liz Doherty
Lillian Liao
Mark Norman
Nobuko Nishimura
Vassil Ognyanov
Dan Retz
Mark Stec
Nuria Tamayo
Phi Tang
Kevin Yang

\section{Process Chemistry TRPV1 Core Team}

Charles Bernard

Chris Borths

Tracy Bostick

Margaret Faul

Andrew Hague

Tony King

Bobby Raihi

Thomas Storz

Jason Tedrow

Oliver Thiel

\section{Collaborators}

Andrej Romanovsky, Andras Garami

St. Joseph's Hospital and Medical Center, Phoenix, AZ

Frank Porreca, Todd Vanderah

University of Arizona, Tucson, AZ

\author{
Narender Gavva \\ Christopher Banfield \\ Anthony Bannon \\ Gudarz Davar \\ Celia Dominguez \\ Margaret Faul \\ Anu Gore \\ David Hovland \\ Pat Kesslak \\ Sonya Lehto \\ Jean-Claude Louis \\ Mark Norman \\ Sekhar Surapaneni \\ James Treanor
}

Narender Gavva

Pain Therapeutics Summit - Oct $7^{\text {th }} 2008$

(C) Narender R. Gavva; Licensee Bentham Open.

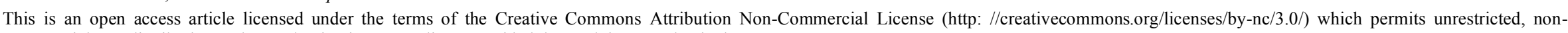
commercial use, distribution and reproduction in any medium, provided the work is properly cited. 